\title{
X-ray Spectroscopy of Cooling Clusters
}

\author{
J. R. Peterson ${ }^{a, 1} \&$ A. C. Fabian ${ }^{\mathrm{b}, 2}$ \\ ${ }^{a}$ Kavli Institute for Particle Astrophysics and Cosmology (KIPAC), Stanford \\ University, PO Box 20450, Stanford, CA 94309, USA \\ ${ }^{\mathrm{b}}$ Institute of Astronomy (IoA), Cambridge University, Madingley Road, \\ Cambridge CB3 OHA, UK
}

\begin{abstract}
We review the X-ray spectra of the cores of clusters of galaxies. Recent high resolution X-ray spectroscopic observations have demonstrated a severe deficit of emission at the lowest X-ray temperatures as compared to that expected from simple radiative cooling models. The same observations have provided compelling evidence that the gas in the cores is cooling below half the maximum temperature. We review these results, discuss physical models of cooling clusters, and describe the X-ray instrumentation and analysis techniques used to make these observations. We discuss several viable mechanisms designed to cancel or distort the expected process of X-ray cluster cooling.
\end{abstract}

1 JRP E-mail: jrpeters@slac.stanford.edu

2 ACF E-mail: acf@ast.cam.ac.uk 


\section{Contents}

\begin{tabular}{lll}
\hline & Introduction & 4
\end{tabular}

2 Clusters of Galaxies 5

\begin{tabular}{lll}
\hline 3 & Phvsics of the Intracluster Medium & 7
\end{tabular}

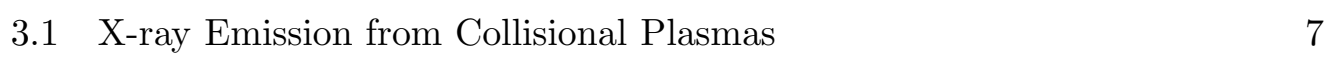

\begin{tabular}{lll}
\hline $3.2 \quad$ Magneto-hvdrodvnamics & 15
\end{tabular}

$\begin{array}{lll}3.3 & \text { Cooling flows } & 18\end{array}$

$4 \quad$ X-rav Instrumentation and Observational Techniques 24

4.1 X-rav Telescopes and their Relevance to Clusters 24

$\begin{array}{lll}4.2 & \text { Analvsis Techniques } & 26\end{array}$

5 X-rav Spectra of Cooling Clusters 28

5.1 Early Work on Imaging Observations 28

5.2 Early Work on Low Resolution Spectroscopv 29

\begin{tabular}{|ll}
5.3 & Focal Plane Crvstal Spectrometer
\end{tabular}

5.4 Reflection Grating Spectrometer Observations 31

5.5 Recent Spatiallv-resolved Spectro-photometric Observations 35

$\begin{array}{lll}5.6 & \text { Observations of Cataclysmic Variables } & 36\end{array}$

5.7 Definition of the Cooling Flow Problem 36

$\begin{array}{lll}6 \quad \text { Are cooling flows ruled out? } & 47\end{array}$

\begin{tabular}{|ll}
6.1 & Cooled gas and star formation
\end{tabular}

\begin{tabular}{lll}
\hline$\quad$ Heating & 50
\end{tabular}

\begin{tabular}{|ll}
\hline $7.1 \quad$ Heat conduction & 51
\end{tabular}

\begin{tabular}{lll}
\hline $7.2 \quad$ Heating by a central radio source & 51
\end{tabular}

$\begin{array}{lll}7.3 & \text { Is the ICM turbulent? } & 57\end{array}$

\begin{tabular}{lll}
\hline $7.4 \quad$ Multiphase flows & 58
\end{tabular} 
7.5 Role of Magnetic Fields and Cosmic Ravs

$7.6 \quad$ Feedback

$7.7 \quad$ Other heat sources and mechanisms

$8 \quad$ Discussions

61

$9 \quad$ Future work

10 Acknowledgments

64 


\section{Introduction}

Observations show that the X-ray emission from many clusters of galaxies is sharply peaked around the central brightest galaxy. The inferred radiative cooling time of the gas in that peak, where the temperature drops to the center, is much shorter than the age of the cluster, suggesting the existence of a cooling flow there (Fabian et al. 1994). X-ray spectroscopy over the past $5 \mathrm{yr}$ shows that the temperature drop toward the center is limited to about a factor of three. Just when the gas should be cooling most rapidly it appears not to be cooling at all. This is sometimes known as the cooling flow problem. Careful observations show that gently distributed heat is required over a radius of up to $100 \mathrm{kpc}$ to balance radiative cooling in these regions.

The issues of cooling and heating of hot gas have broad relevance to the gaseous part of galaxy formation and evolution. Brightest cluster galaxies (BCG) are the most massive galaxies known. Calculations of the clustering behaviour of cold dark matter predict a power-law mass distribution for large galaxies whereas the stellar mass observed has an exponential distribution (Benson et al. 2003). The truncation of the stellar mass distribution in massive galaxies is likely due to the process which stops cooling flows. Simple cooling flows are an ingredient of semi-analytical models for galaxy formation. The cooling of hot gas to form stars is essential for the growth of massive galaxies and cannot be studied directly for isolated systems due to Galactic absorption. The cores of galaxy clusters offer examples which can be directly observed. However they do not appear to operate in any simple manner. The problem appears to be widespread, from the most massive clusters to the centers of individual elliptical galaxies. Heating and cooling problems of hot gases are common in astronomy, with examples ranging from the interstellar medium of our own Galaxy to the Solar Corona.

The diffuse hot ionized plasma in clusters is magnetized which means that MHD processes may be important (Schekochihin et al. 2004).

Here we briefly review the main X-ray properties and emission processes of the intracluster medium (ICM) before showing the X-ray spectra of cool cores. We then discuss the main solutions which have been proposed for the cooling flow problem. 


\section{Clusters of Galaxies}

Clusters are the most massive bound and quasi-relaxed objects in the Universe. They have total masses of $10^{14}$ to above $10^{15} \mathrm{M}_{\odot}$. The total gas fraction is about 16 per cent with about 13 per cent in the hot ICM and the remaining 3 per cent in stars in the cluster galaxies. The remaining 84 per cent of the mass is in dark matter. Gas densities in cluster centers range from as much as $10^{-1} \mathrm{~cm}^{-3}$ in peaked clusters to $10^{-3} \mathrm{~cm}^{-3}$ in the non-peaked ones. This is in stark contrast to the mean cosmic density of baryons of about $10^{-8} \mathrm{~cm}^{-3}$.
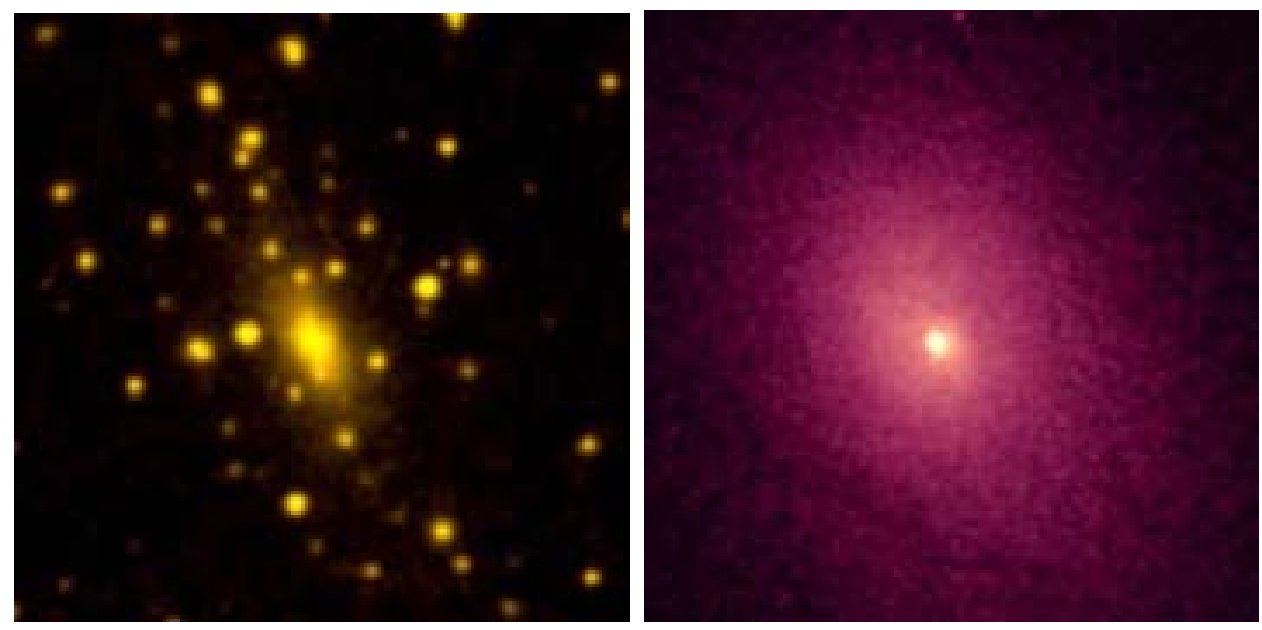

Fig. 1. Chandra X-ray (left) and DSS optical (right) image of the relaxed massive galaxy cluster, Abell 2029. Both images are 4 arcminutes on a side. Abell 2029 is an extremely regular and putative cooling flow cluster. The X-ray image demonstrates how the intracluster medium pervades the space between the galaxies shown in the optical image. Figure adapted from http://www.chandra.harvard.edu/ (X-ray: NASA/CXC/UCI/A.Lewis et al. Optical: Pal.Obs. DSS).

The characteristic or virial radius, $R_{\mathrm{v}}$, of a cluster, defined from the theory of structure collapse in an expanding universe as where the mean density of the cluster is 200 times the critical density of the Universe (i.e. $200 \times 3 H^{2} / 8 \pi G$, with the Hubble constant at redshift $z$ varying as $\left.H / H_{0}=\sqrt{\Omega_{\mathrm{m}}(1+z)^{3}+1-\Omega_{\mathrm{m}}}\right)$, is typically between 1 and $3 \mathrm{Mpc}$. The gas is heated by gravitational infall to temperatures close to the virial temperature $k T \sim G M m_{\mathrm{p}} / R_{\mathrm{v}}$, which ranges in clusters from $1-15 \mathrm{keV}$. The total X-ray luminosities range from about $10^{43} \mathrm{erg} \mathrm{s}^{-1}$ to $10^{46} \mathrm{erg} \mathrm{s}^{-1}$. Objects at lower masses and luminosities are groups which have from a few to tens of member galaxies as compared with the hundreds of galaxies in a typical cluster.

Structure formation in the Universe proceeds in a hierarchical manner with the most massive objects, clusters, forming last, which means now. They continue to evolve by the infall of subclusters. The time since the last major merger is typically about 5 Gyr. About 20 per cent of clusters have had a more recent 
merger or are undergoing one. These are not the subject of this review.

Analytic and numerical simulations of cluster formation indicate that the total X-ray luminosity $L_{\mathrm{X}} \propto T^{2}$ in the absence of gas cooling and heating. This follows since the X-ray luminosity is dominated by thermal bremsstrahlung so $L_{\mathrm{X}} \propto n^{2} T^{1 / 2} R_{\mathrm{v}}^{3}$, the mean gas density $n \propto \frac{M}{R_{\mathrm{v}}^{3}}$ is constant and $T=\frac{M}{R_{\mathrm{v}}}$. The temperature drops monotonically outward (by a factor of up to about 2 ). Observations instead show $L_{\mathrm{X}} \propto T^{3}$ over the temperature range $2-8 \mathrm{keV}$ with a wide dispersion at lower temperatures and a possible flattening above. The simplest explanation for this result is that the gas has had additional heating of 2-3 keV per particle (Wu et al. 2000; Voit et al. 2003). The effect of such heating is not to increase the temperature by that amount but mostly to expand the gas (reducing its density and thus X-ray luminosity). Such energy is plausibly due to energy output from active galaxies, i.e. accreting black holes in cluster galaxies. Alternatively, radiative cooling by removing the lowentropy gas in star formation may reproduce the relation as well (Voit \& Brvan 2001).

The gas has generally been enriched to 0.3 of the Solar value by early supernovae. In relaxed clusters the potential and gas peak on the BCG. The metallicity often rises to solar or even higher around the BCG, probably due to SN Ia.

In relaxed, X-ray peaked, clusters the temperature profile is often inverted in the inner core (i.e. $R<100 \mathrm{kpc}$ ) dropping inward as $T \propto r^{\alpha}$ with $\alpha \sim 0.3-0.5$. The gas density there rises as $n \propto r^{-1}$.

The overall profiles of the gas density and temperature depend on the entropy of the gas and thus on its heating and cooling history, subject to the equation of hydrostatic equilibrium,

$$
\frac{d p}{d r}=-\rho g \text { or } \frac{d(n k T)}{d r}=-n \mu m_{\mathrm{p}} \frac{G M(<r)}{r^{2}} .
$$

where $p$ is the pressure, $\rho$ is the mass density, $n$ is the number density, $k$ is Boltzmann's constant, $T$ is the temperature, $G$ is Newton's constant, $g$ is the gravitational acceleration, $M(<r)$ is the enclosed mass within a radius $r, m_{P}$ is the proton mass, and $\mu m_{p}$ is the mean mass per particle. This equation is used to estimate the total mass profile of clusters. Massive ones can act as gravitational lenses for background galaxies as seen in the optical band which provides another means to measure mass profiles. Agreement between profiles determined by both methods (Allen et al. 2001d) show that hydrostatic equilibrium holds well in the main body of relaxed massive lensing clusters and that any non-thermal pressure there is not dominant. 


\section{Physics of the Intracluster Medium}

The intracluster medium (ICM) is plasma that is nearly fully ionized due to the high temperatures created by the deep dark matter gravitational potential. Hydrogen and helium, for example, are fully stripped of their electrons. Heavier elements have retained only a few of their electrons in this hot medium. In addition to free electrons and ions in the plasma, electromagnetic radiation, which is emitted mostly as X-rays, is created by quantum mechanical interactions in the plasma.

The physics of the ICM can be studied as two physical phenomena: 1) the ionized plasma, and 2) the radiation emission processes. The ionized plasma is well-described by magneto-hydrodynamic theory on large enough scales. The radiation emission processes are governed by equations for X-ray emission from a collisionally-ionized plasma. We can treat these phenomena separately because the intracluster medium is mostly optically-thin, (i.e. the radiation almost completely escapes without interacting with the plasma). Later in the next section, we will show that this is the case.

In $§ 3.1$, we describe how X-rays are produced in the intracluster medium. In section $\S 3.2$, we motivate magneto-hydrodynamic theory as the description of the plasma. In $\S 3.3$, we unify the two theoretical descriptions to produce what we refer to as the "standard cooling-flow model". This model will serve as the basis for the physical description of the cores of clusters of galaxies. In later sections, we describe the puzzling observations that seem to agree with many expectations of this model, but disagree strongly with other aspects of the model.

\subsection{X-ray Emission from Collisional Plasmas}

Ionized plasmas produce copious amounts of X-rays. The emission of X-rays has two important consequences. First, it allows us to observe the intracluster medium by detecting those X-rays. Furthermore, since most of those X-rays do not interact between their emission and their detection in X-ray telescopes it allows us to study the intracluster medium in an unperturbed state. Through $\mathrm{X}$-ray spectroscopy and imaging, we can measure several physical quantities, such as the temperature and density, at various positions in the cluster.

The second important consequence is that the emission of these X-rays will tend to cool the plasma. A significant quantity of energy is carried away by the $\mathrm{X}$-rays as they escape the cluster. The emission of these $\mathrm{X}$-rays was thought to set up a non-linear process of excessive cooling in cluster cores that is broadly termed a "cooling flow", a major subject of this work. In the following, we 
describe the process of X-ray emission from collisionally-ionized plasmas and show how it relates to the intracluster medium.

\subsubsection{Coronal Approximation}

The emission of X-rays from ionized atoms in a plasma can be quite complex. Fortunately, we can make use of several approximations to simplify the emission processes. The emission of X-rays in the ICM is well-described by the coronal approximation (Elwert 1952; Mewe 1999). These approximations describe optically-thin plasmas in collisional equilibrium. Collisional equilibrium occurs when electron collisional ionization processes are balanced precisely by recombination processes. The coronal approximation, as the name implies, was originally developed for study of the Solar Corona, but the condition also applies to gas in clusters of galaxies, as well as hot gas in elliptical, starburst galaxies, and older supernovae remnants. In this approximation, there are three important conditions that specify the thermodynamic state of the free electrons, ions, and photons in the plasma, as well as the electron distribution within each ion.

The first approximation is that the photons are assumed to be free and do not interact with either the electrons or the ions after they are created. This has the importance consequence that photo-ionization processes (ionizing atoms by photons) and photo-excitation processes (raising an electron in an atom to an excited level) are far less frequent that electron collisional ionization and excitation processes. The radiation densities are low enough in clusters of galaxies for this condition to be met, except possibly for resonant scattering in some strong emission lines with high oscillator strength (Gilfanov et al. 1987).

The second approximation is that the atoms can be treated as if their electrons are all in their ground state rather than having a Boltzmann distribution as is common in LTE (local thermodynamic equilibrium) gases. This is true if there is a low enough electron and radiation density, so that the excitations that are density dependent are less frequent than the radiative decays, which are quite fast for X-ray energies. The radiation density is low as discussed above. This condition is also met for electron densities below $10^{10} \mathrm{~cm}^{-3}$ for even slowly decaying metastable states. Densities in clusters are at most $10^{-1} \mathrm{~cm}^{-3}$.

The final approximation is that the plasma is locally relaxed to a Maxwellian distribution around a common electron temperature, T. The free electrons and ions are assumed to have obtained a common temperature. This is only valid if typical dynamical time-scales, such as the time it takes the plasma to cool, is much longer than the time scale for sharing energy between electrons and ions, such as the electron recombination time scale or time scale between Coulomb collisions. If this is true, then collional equilibrium is achieved in 
which ionizations are balanced by recombinations. This assumption is valid in the cores of cluster, but may break down in the outer regions of clusters where the density is lower and the plasma may still be in the process of ionizing. One might also worry that this assumption may break down in the cores of clusters if there is a complex multi-phase distribution of plasma temperatures due to thermally unstable pockets of plasma each with their own temperature. Generally if such a situations exists, however, it is likely that each pocket will locally achieve a common temperature. An electron penetrating a cloud of a different temperature will interact and achieve a Maxwellian distribution. It is only with complex and rapidly mixed interfaces that the time scale of injection of a new cloud is much shorter than $\frac{\lambda_{e}}{v_{e}}$, where $v_{e}$ is the electron velocity, and $\lambda_{e}$ is the electron mean free path, that the collisional equilibrium would be violated.

\subsubsection{Ionization Balance}

The fraction of atoms at a given charge state is determined by a balance of ionizations and recombinations. The exact balance is fixed by a coupled set of differential equations that relate the ionization and recombination processes between neighboring charge states. The equations are of the form,

$$
\frac{d n_{i}}{d t}=-I_{i} n_{e} n_{i}-R_{i} n_{e} n_{i}+I_{i-1} n_{e} n_{i-1}+R_{i+1} n_{e} n_{i+1}
$$

where $n_{e}$ is the electron number density, $n_{i}$ is the density of atoms in the $i$ th charge state, $I_{i}$ is the ionization rate out of the $i$ th charge state, and $R_{i}$ is the recombination rate out of the $i$ th state. We have ignored spatial diffusion of the ionization balance. Note, that it is customary to express the density of a given ion relative to the number density of hydrogen, $n_{H}$. We then define $a_{i}$ as the relative abundance and the fraction of atoms in a given charge state as $f_{i}$. Then we have, $n_{i}=n_{H} f_{i} a_{i}$, where $n_{H} a_{i}$ drops out of the above equations. Collisional equilibrium assumes that a steady-state has been achieved, so that the left hand side is set to zero. The equations therefore simplify to equations of the form,

$$
0=-I_{i} f_{i}+R_{i+1} f_{i+1}
$$

Collisional equilibrium will eventually be achieved if a plasma remains undisturbed for a long enough period of time (i.e., the inverse of the recombination rate). Several calculations have been done to determine this ionization balance

as a function of the electron temperature (Jordan 1969; Arnaud \& Rothenflug 1985; Arnaud \& Ravmond 1992; Mazzotta 1998). One such calculation is shown in Figure 2. 


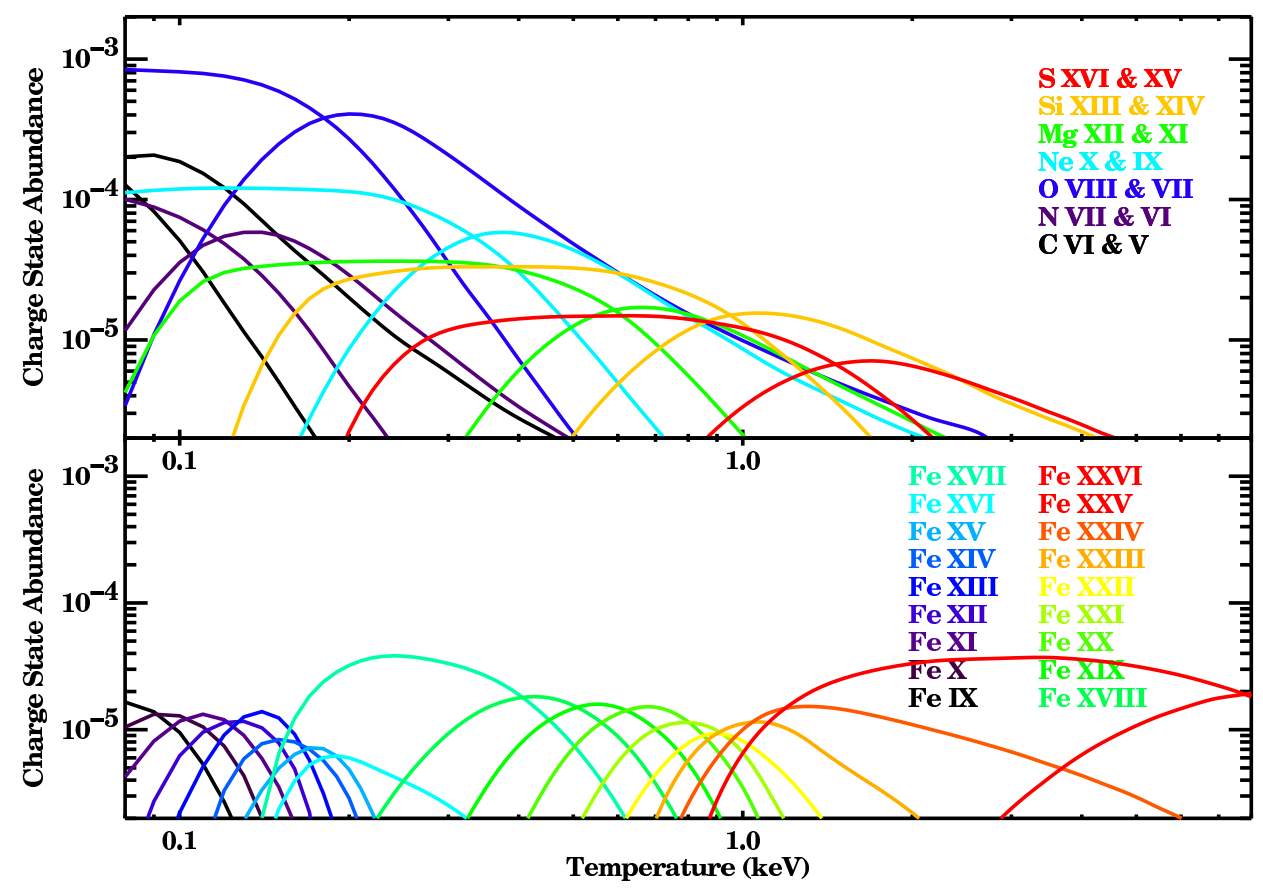

Fig. 2. The charge state abundance (elemental abundance times fraction ionic abundance) of various ions as a function of temperature. The top panel shows helium-like and hydrogen-like charge states of various low $\mathrm{Z}$ atoms. The bottom panel shows iron ions having the outer electron in the $\mathrm{K}, \mathrm{L}$, and $\mathrm{M}$ shell. The bottom panel indicates how the measurement of various ions in the iron series is a sensitive probe of whether plasma at a given temperature exists. Figure uses data from Arnaud \& Ravmond 1992.

Once the ionization balance is determined the X-ray spectrum can be calculated by considering the various radiation processes. The most important processes are bremsstrahlung and the $\mathrm{K}$ and $\mathrm{L}$ shell transitions for the discrete line emission.

\subsubsection{Bremsstrahlung and other Continuum Processes}

When Hydrogen is ionized above temperatures of $2 \times 10^{4} \mathrm{~K}$, copious amounts of bremsstrahlung emission are produced. Bremsstrahlung radiation results from the accelerations of the free electrons in the Coulomb field of an ion. The spectrum is roughly independent of energy below the energy equal to $k T_{e}$, where $T_{e}$ is the electron temperature and $k$ is Boltzmann's constant. A rough approximation of the power per energy per volume radiated by bremsstrahlung is given by the equation below,

$$
\frac{d^{2} P}{d V d E} \approx 10^{-11} n_{e} n_{H} T^{-\frac{1}{2}} e^{-\frac{E}{k T}} \mathrm{~cm}^{-3} \mathrm{~s}^{-1}
$$


where $n_{e}$ is the electron density, $n_{H}$ is the hydrogen density, $E$ is the photon energy, $k$ is Boltzmann's constant, and $T$ is the electron temperature in Kelvin. The total power radiated is therefore,

$$
\frac{d P}{d V} \approx 10^{-27} n_{e} n_{H} T^{\frac{1}{2}} \operatorname{ergs~} \mathrm{cm}^{-3} \mathrm{~s}^{-1}
$$

In addition to bremsstrahlung, bound-free emission (the capture of a free electron to a bound state) and two-photon emission (which occurs most frequently following a collisional excitation of Hydrogen to the 2 s level) are also significant source of continuum radiation. They both modify the shape of the continuum emission.

\subsubsection{Discrete Line Emission}

Discrete line emission is formed by a number of atomic processes. Such line emission is the most important tool for the X-ray spectroscopist. The most important atomic processes are collisional excitation, radiative recombination, dielectronic recombination, and resonant excitation. Generally, the processes have been incorporated in a number of publically available and well-tested codes that are used to study collisionally-ionized spectra.

The strength of an emission line is determined by the excitation and recombination rates, which are proportional to the integral of the velocity times the cross-section for a particular process over a Maxwellian distribution. The volume emissivity for a given emission line is calculated by equations of the form,

$$
\epsilon=n_{e} n_{i}\left(C^{I I} f_{i-1}+C^{E} f_{i}+\alpha^{R} f_{i+1}\right)
$$

where $C^{I I}$ is the rate of inner shell ionization processes, $C^{E}$ is the sum of collisional excitation processes, and $\alpha^{R}$ is the sum of recombination processes. Several well tested codes have been developed to calculate the emergent spectrum by assuming an ionization calculation and including a set of excitation and recombinations rates. These include the Raymond-Smith (Ravmond \& Smith 1977), MEKA (Mewe et al. 1985, 1986; Kaastra 1992; Mewe et al. 1995), MEKAL (Liedahl et al. 1995), and APEC codes (Smith et al. 2001). These codes are compilations of the results of more detailed atomic codes, which solve the Dirac equation either by the distorted wave approximation (Bar-Shalom et al. 2001; Gu et al. 2003) or R-matrix methods (Berrington et al. 1995). The number of transitions and the accuracy of the detailed processes limits the results. Extensive laboratory work has been applied to verify wavelengths (Brown et al. 1998, 2002) and cross-sections ( $\mathrm{Gu}$ et al. 1999; Chen et al. 2005) of the transitions. 
A number of common spectral transitions occur in most X-ray spectra. In fact, despite the complexity implied by the above discussion there are usually only a couple of dozen strong transitions that are used to determine most of the information that can be extracted from the X-ray spectrum. Several of the important emission line blends are shown in Table 1.

\subsubsection{X-ray Cooling Function and Emission Measure Distribution}

The X-ray cooling function is calculated by integrating the emission from all processes and weighting by the energy of the photons.

$$
\Lambda\left(T, Z_{i}\right)=\int_{0}^{\infty} d E E \frac{d \alpha}{d E}\left(E, T, Z_{i}\right)
$$

where $\frac{d \alpha}{d E}$ is the energy dependent line power (or continuum power). The cooling function relates the total amount of energy emitted per volume for a given amount of plasma with a given temperature and emissivity. The cooling function has been compiled in various tables (Böhringer \& Hensler 1992; Sutherland \& Dopita 1993).

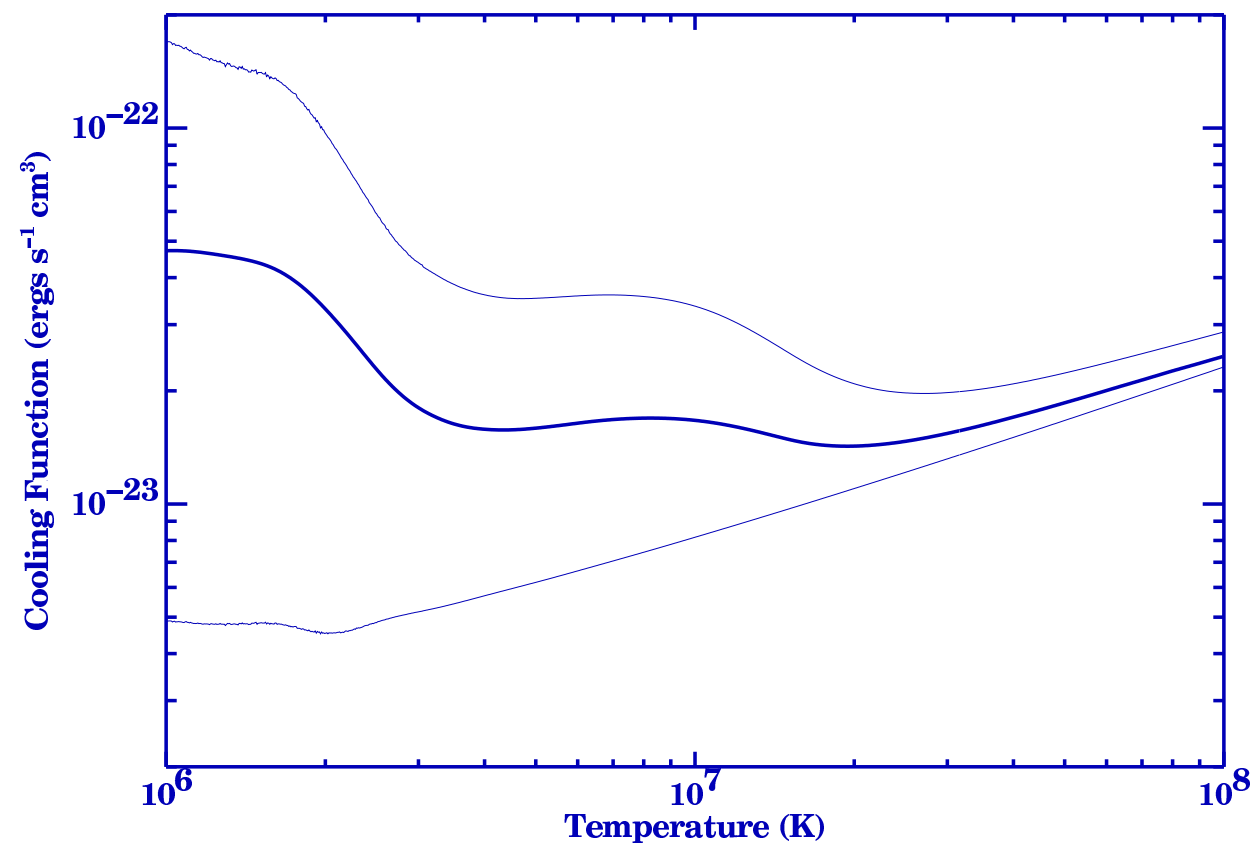

Fig. 3. The radiative cooling function is shown for solar abundances (top curve), one-third solar abundances (middle), and pure Hydrogen and Helium (bottom). The $\mathrm{X}$-ray region from $10^{6}$ to $10^{8} \mathrm{~K}$ is dominated by bremsstrahlung at high temperatures as well as significant contribution from line emission at lower temperatures. Below $10^{6} \mathrm{~K}$ in the UV temperature range, the cooling function rises significantly. 
Table 1

Important line blends in Cluster X-ray Spectra

\begin{tabular}{|c|c|c|c|}
\hline $\operatorname{Ion}^{a}$ & Wavelengths & Energies & Temperature \\
\hline & $\AA$ & $\mathrm{keV}$ & $\mathrm{keV}^{\mathrm{b}}$ \\
\hline Fe XXVI & 1.8 & 6.97 & $>3.0$ \\
\hline Fe XXV & $1.9,1.9$ & $6.70,6.63$ & $1.0 \rightarrow 8.0$ \\
\hline Fe XXIV & $10.6,11.2$ & $1.17,1.11$ & $0.9 \rightarrow 4.0$ \\
\hline Fe XXIII & $11.0,11.4,12.2$ & $1.13,1.09,1.02$ & $0.8 \rightarrow 2.0$ \\
\hline Fe XXII & $11.8,12.2$ & $1.05,1.02$ & $0.6 \rightarrow 1.5$ \\
\hline Fe XXI & $12.2,12.8$ & $1.02,0.97$ & $0.5 \rightarrow 1.0$ \\
\hline Fe XX & $12.8,13.5$ & $0.97,0.92$ & $0.4 \rightarrow 1.0$ \\
\hline Fe XIX & $13.5,12.8$ & $0.92,0.97$ & $0.3 \rightarrow 0.9$ \\
\hline Fe XVIII & $14.2,16.0$ & $0.87,0.77$ & $0.3 \rightarrow 0.8$ \\
\hline \multirow[t]{2}{*}{ Fe XVII } & $15.0,17.1$ & $0.83,0.73$ & $0.2 \rightarrow 0.6$ \\
\hline & $15.3,16.8$ & $0.81,0.73$ & \\
\hline S XXVI & 4.7 & 2.62 & $>1.0$ \\
\hline S XXV & $5.1,5.0$ & $2.43,2.46$ & $0.3 \rightarrow 1.0$ \\
\hline Si XIV & 6.2 & 2.00 & $>1.0$ \\
\hline Si XIII & $6.6,6.7$ & $1.87,1.84$ & $0.2 \rightarrow 1.0$ \\
\hline Mg XII & 8.4 & 1.47 & $>0.7$ \\
\hline Mg XI & $9.2,9.3$ & $1.35,1.33$ & $0.1 \rightarrow 0.6$ \\
\hline Ne X & 12.2 & 1.02 & $>0.4$ \\
\hline Ne IX & $13.5,13.7$ & $0.92,0.90$ & $0.1 \rightarrow 0.3$ \\
\hline O VIII & $19.0,16.0$ & 0.64 & $>0.2$ \\
\hline O VII & $21.6,22.0$ & $0.57,0.56$ & $0.1 \rightarrow 0.2$ \\
\hline N VII & 24.8 & 0.50 & $>0.1$ \\
\hline C VI & 33.7 & 0.37 & $>0.1$ \\
\hline
\end{tabular}

a Note this line transition list is somewhat crude since we have chosen to tabulate line blends rather than actual transitions, but matches well with the quality of the observations.

$b$ The temperature range is calculated to roughly show the temperature range where the emissivity of a given ion blend is within an order of magnitude of its peak emissivity. 
The relative distribution of plasma at a set of temperatures is often expressed in terms of an emission measure distribution. The differential emission measure, $\frac{d E M}{d T}$ is defined by

$$
\frac{d \epsilon}{d E}=\int_{0}^{\infty} \frac{d E M}{d T} \frac{d \alpha}{d E}(E, T) d T
$$

where $\frac{d \epsilon}{d E}$ is the energy-dependent emissivity and the integral is over all temperatures. It is also convenient to express the distribution of plasma temperatures in terms of the differential luminosity, which is defined by

$$
\frac{d L}{d T}=\frac{d E M}{d T} \Lambda(T)
$$

\subsubsection{Cooling Time}

The cooling time of an optically-thin plasma is the gas enthalpy divided by the energy lost per unit volume of the plasma. The gas enthalpy is $\frac{5}{2} n k T$ and the energy lost per volume is the electron density squared times the cooling function. The cooling time can then be written as,

$$
t_{\text {cool }} \equiv \frac{\frac{5}{2} n k T}{n^{2} \Lambda} \approx t_{H} T_{8} \Lambda_{-23}^{-1} n_{-2}^{-1}
$$

where $t_{H}$ is the age of the universe $(13.7 \mathrm{Gyr}), T_{8}$ is the temperature in units of $10^{8} \mathrm{~K}, \Lambda_{-23}$ is the cooling function in units of $10^{-23} \mathrm{ergs}^{3} \mathrm{~cm}^{-1}$, and $n_{-2}$ is the density in units of $10^{-2}$ particles $\mathrm{cm}^{-3}$. We used the gas enthalpy per volume, $\frac{5}{2} n k T$ instead of the thermal energy per volume $\frac{3}{2} n k T$ since the plasma is compressed as it cools which therefore effectively raises its heat capacity by a factor of $\frac{5}{3}$. Therefore, X-ray plasma with gas density above $10^{-2} \mathrm{~cm}^{-3}$ has had sufficient time to cool. In the cores of cooling clusters the cooling time approaches cooling times below $5 \times 10^{8}$ yr. If the gas was undisturbed, it would have a chance to cool several times. Note that as gas cools at constant pressure (due to the weight of overlying gas) then the rise in density as the temperature drops means that $t_{\text {cool }}$ becomes shorter and shorter.

\subsubsection{Optical Depth, Resonance Scattering, and Opacity}

The optical depth for photons of a given wavelength, $\tau$, is the product of the column density of a particular ion, $N_{i}$ and the cross-section of a particular process. The cross-section is a function of energy. The column density is the line integral of the ion density, $n_{i}$, which is a function of the spatial position along the line of sight. 
The intracluster medium is, for the most part, optically-thin to its own radiation (i.e. photons escape once they are emitted without interacting again). Photons with energies close to certain resonance transitions, however, can scatter several times before leaving a cluster. The optical depth to a resonance transition is given by

$$
\tau=\int d \ln _{i} f_{i} \frac{\pi e^{2}}{m_{e} c} \frac{\exp ^{-\frac{\left(\lambda-\lambda_{i}\right)^{2}}{2 \sigma_{i}^{2}}}}{\sqrt{2 \pi \sigma_{i}^{2}}}
$$

where $n_{i}$ is the ion density, $f_{i}$ is the oscillator strength of the transition, $m_{e}$ is the electron mass, $\lambda_{i}$ is the wavelength of the transition, $c$ is the speed of light, $e$ is the electron charge, and $\sigma_{i}$ is the line width given by,

$$
\sigma_{i}=\lambda_{i} \sqrt{\frac{k T}{\left(\frac{A}{1+\frac{5}{3} M^{2} \frac{A}{\mu}}\right) m_{p} c^{2}}}
$$

where $A$ is the atomic mass number, $\mu$ is the mean mass per particle, $M$ is the Mach number of the turbulence or gas motions in the plasma, $k$ is Boltzmann's constant, and $T$ is the temperature. The above expression includes both the thermal broadening of the line as well as turbulent broadening.

If a significant quantity of lowly ionized matter exists along the line of sight, X-rays can be absorbed and re-emitted at lowly ionized longer wavelengths. This situation occurs frequently for absorption from neutral gas in the Milky Way Galaxy, but could also occur from gas trapped in the cluster potential. This subject is discussed in detail later, but we note that understanding the role of absorption often has a significant effect on the interpretation of the soft X-ray spectrum. This is particularly true at low resolution. Helium K shell absorption at low energies and Oxygen $\mathrm{K}$ shell absorption at $23.5 \AA$ are the largest contributors to the opacity from a neutral absorber and produce absorption edge features in the spectrum.

\subsection{Magneto-hydrodynamics}

The plasma of the intracluster medium can be described by magneto-hydrodynamics on large scales. Several assumptions underlie this statement. First, the ICM is optically-thin so we do not have to include radiative forces in the theory. Second, we assume the ICM is non-relativistic. This is true since the sound speed is at most a few thousand kilometers per second. Third, we assume the ICM is nearly fully-ionized, which is true since Hydrogen consitutes most of the plasma. Finally, the plasma can be treated as a fluid using continuous 
fields if the plasma parameter is small so that collective processes dominate. The plasma parameter is defined by

$$
g \equiv \frac{1}{n \lambda_{D}^{3}}=8 \times 10^{-3} n^{\frac{1}{2}} T^{-\frac{3}{2}}
$$

where $\lambda_{D}$ is the Debye length, $n$ is the density in units of $\mathrm{cm}^{-3}$, and $T$ is the temperature in $\mathrm{K}$. The ICM plasma parameter is in fact extremely small.

Magnetohydrodynamics (MHD) in this context is described by the following variables: the fluid velocity, $\mathbf{v}$, the temperature, $T$, the density, $\rho$, and the magnetic field, $\mathbf{B}$, and the dark matter density, $\rho_{D M}$. These quantities are related to one another by the full set of MHD equations, which assume local thermodynamic equilibrium of these quantities. The transport properties of the fluid can be written in terms of the viscosity, $\eta$, conductivity, $K$, and resistivity, $\lambda$.

We also adopt the following notation. $T$ is the energy per particle; whereas $T_{K}$ is the temperature in Kelvin. They are related to one another by the relation, $T=k T_{K} /\left(\mu m_{p}\right) . k$ is Boltzmann's constant, the mass of the proton is $m_{p}$, and the mean mass per particle is $\mu m_{p}$. Additionally, the cooling luminosity is usually defined in terms of the electron, $n_{e}$ and hydrogen, $n_{H}$, number densities, which are related to the fluid mass density by, $n_{e}=n_{H} / 1.19=$ $\rho /\left(\mu m_{p}\right)$.

Below are the 5 MHD equations. 


$$
\begin{gathered}
\frac{\partial \rho}{\partial t}+\nabla \cdot(\rho \mathbf{v})=0 \\
\rho \frac{\partial v_{i}}{\partial t}+\rho(\mathbf{v} \cdot \nabla) v_{i}=\rho \frac{\partial \Phi}{\partial x_{i}}-\frac{\partial}{\partial x_{i}}\left(\rho T+\frac{B^{2}}{8 \pi}\right)+\frac{(\mathbf{B} \cdot \nabla) B_{i}}{4 \pi} \\
+\frac{\partial}{\partial x_{j}}\left[\eta\left(\frac{\partial v_{i}}{\partial x_{j}}+\frac{\partial v_{j}}{\partial x_{i}}-\frac{2}{3} \delta_{i j} \nabla \cdot \mathbf{v}\right)\right] \\
\frac{\partial \mathbf{B}}{\partial t}=\nabla \times(\mathbf{v} \times \mathbf{B})-\nabla \times(\lambda \nabla \times \mathbf{B}) \\
\frac{3 T}{2} \frac{\partial T}{\partial t}+\frac{3}{2} \rho \mathbf{v} \cdot \nabla T+\rho T \nabla \cdot \mathbf{v}=\nabla \cdot\left(K \nabla T_{K}\right)+\frac{\lambda}{4 \pi}(\nabla \times \mathbf{B}) \cdot(\nabla \times \mathbf{B}) \\
+2 \eta\left(\frac{\partial v_{i}}{\partial x_{j}}+\frac{\partial v_{j}}{\partial x_{i}}\right)^{2}-\frac{2}{3} \eta(\nabla \cdot \mathbf{v})^{2}-n_{e} n_{H} \Lambda(T, Z)+\text { interactions } \\
\nabla^{2} \Phi=4 \pi G\left(\rho+\rho_{D M}\right)
\end{gathered}
$$

The first equation, the mass conservation equation, is a statement that the total mass of the fluid is constant. The second equation, the Navier-Stoker equation, enforces momentum conservation. This equation relates the momentum of the fluid (left hand side) to the gravitational compression (first term on the right hand side), the thermal and magnetic pressure gradients (second and third term), tangetial magnetic transport (fourth term), and viscous forces (last terms).

The evolution of the magnetic field follows the induction equation, the third equation. The first term on the right hand side generates the magnetic field due to plasma motion, and the final term dissipates the magnetic flux due to magnetic reconnection.

The energy equation, the fourth equation, expresses the balance between heating and cooling terms. The left hand side describes the energy content of the plasma (first and second term) as well as its compression (third term). The right hand side contains a conduction term (first term), a magnetic dissipation term (second term), viscous heating terms (third and fourth term), and the energy lost due to radiative cooling. In addition, this equation could include interactions between this plasma and other matter, such as dust, cosmic rays, or dark matter. Finally, the gravitational field is set by the fifth equation where both the dark matter and plasma contribute to the gravitational field. 
The transport coefficients for the intracluster medium is the subject of much theoretical work. The values of these coefficients for a ionized plasma with no magnetic field was worked out by Spitzer et al. (1962) using kinetic theory. These values are given by:

$$
\begin{aligned}
K & \approx 10^{-5} T^{5 / 2} \mathrm{ergs} \mathrm{cm}^{-1} \mathrm{~s}^{-1} \mathrm{deg}^{-1} \\
\eta & \approx 10^{-17} T^{5 / 2} \mathrm{~g} \mathrm{~cm}^{-1} \mathrm{~s}^{-1} \\
\lambda & \approx 7 \times 10^{12} T^{-3 / 2} \mathrm{~cm}^{2} \mathrm{~s}^{-1} .
\end{aligned}
$$

A tangled magnetic field due to MHD turbulence, e.g. Goldreich \& Sridhar 1995, could modify these coefficient considerably and there has been considerable debate in this subject (Tao 1995; Chandran \& Cowlev 1998; Naravan \& Medvedev 2001; Maron et al. 2004). We will return to this subject in later sections.

\subsection{Cooling flows}

The radiative cooling time in the cores of at least two thirds of low redshift (Peres et al. 1998) and moderate redshift (Bauer et al. 2002) clusters is less than 10 Gyr and for one third it is less than about 3 Gyr. The energy loss is directly due to the observed X-ray emission with no major bolometric correction. If there is no heating to compensate the cooling then a cooling flow occurs in these regions. In order to understand what the 'cooling-flow problem' is, why heating is required, how a 'residual flow' might operate and what happens when heating is not operating, we now briefly examine cooling flows.

\subsubsection{Single-phase flows}

The radiative cooling time $t_{\text {cool }}$ at tens of $\mathrm{kpc}$ radius in a cluster always exceeds the gravitational dynamical time so cooling leads to a slow, subsonic inflow there. The flow causes the density to rise and so maintain the pressure, which is determined by the weight of the overlying gas.

We can simplify the MHD equations significantly for the simple case of an unmagnetized single-phase subsonic flow. We also ignore any terms with conduction, viscosity, and resistivity. If we simplify the previous equations and rewrite the LHS of the energy equation based on the mass equation, 


$$
\begin{gathered}
\frac{\partial \rho}{\partial t}+\nabla \cdot(\rho \mathbf{v})=0 \\
\rho \frac{\partial v_{i}}{\partial t}+\rho(\mathbf{v} \cdot \nabla) v_{i}=\rho \frac{\partial \Phi}{\partial x_{i}}-\frac{\partial(\rho T)}{\partial x_{i}} \\
\rho\left[\left(\frac{\partial}{\partial t}+\mathbf{v} \cdot \nabla\right) \frac{5}{2} T-\frac{1}{\rho}\left(\frac{\partial}{\partial t}+\mathbf{v} \cdot \nabla\right) p\right]=-n_{e} n_{H} \Lambda(T, Z)+\operatorname{interactions}(24) \\
\nabla^{2} \Phi=4 \pi G\left(\rho+\rho_{D M}\right) .
\end{gathered}
$$

We now assume a spherical geometry and assume that the system is in a steady-state such that the partial time derivatives can be ignored, and assume that the flow is subsonic, then terms of order $v^{2}$ can be ignored. We combine the second equation with the third, and define $\dot{M}$ in the first equation, and obtain,

$$
\begin{gathered}
\dot{M} \equiv 4 \pi r^{2} \rho v=\text { constant } \\
\rho \frac{d \Phi}{d r}=\frac{d(\rho T)}{d r} \\
\rho v \frac{d}{d r}\left(\frac{5}{2} T-\Phi\right)=-n_{e} n_{H} \Lambda(T, Z)+\text { interactions } \\
\frac{1}{r^{2}} \frac{d}{d r}\left(r^{2} \frac{d \Phi}{d r}\right)=4 \pi G\left(\rho+\rho_{D M}\right) .
\end{gathered}
$$

When the gravitational field is important, then the temperature approaches a critical solution which 'follows the gravitational potential' (Fabian et al. 1984; Nulsen 1986) as can be seen from the hydrostatic equation for a power law solution. Essentially the flow settles down to a temperature profile close to the local virial one. In an NFW (Navarro et al. 1997) potential where the inner power-law part has dark matter mass density varying as $\rho \propto r^{-1}$ this means $T \propto r$. The temperature then flattens to $T \sim 1 \mathrm{keV}$ within the inner $\approx 10 \mathrm{kpc}$ where the gravitational potential of the central galaxy, assume isothermal, dominates. The temperature finally collapses at the center, before which the flow may go supersonic (the inertial velocity term is then needed in the above momentum equation). Over most of the region $r / v \approx t_{\text {cool }}$, as seen in the energy equation, which varies as $T^{\frac{1}{2}} / n$ for bremsstrahlung. Using the mass flow equation to substitute for $v, n \propto r^{-\frac{5}{4}}$ in the NFW case which leads to a steeper surface brightness profile (the emissivity is proportional to $n^{2}$ ) than observed. 
Even with the King potential commonly used before the 1990s, it was realized that the central surface brightness was too steep to match the observed profiles (Fabian et al. 1984). Interpreted as a cooling flow, the data indicated that the mass flow rate increased with radius (roughly as $\dot{M} \propto r$ out to the cooling radius $r_{\mathrm{c}}$ where $t_{\text {cool }} \sim 10^{10} \mathrm{yr}$ ). Such a situation requires that matter is cooling out over a range of radii, which was explained by gas with a range of densities and thus cooling times occurring at a given radius. Therefore, more generic models than a simplified spherical single phase flow had to be considered.

\subsubsection{Thermal Instability and Multiphase flows}

The above discussion of single phase flows says little about what happens on small scales. In particular, when cooling begins and what size perturbation leads to the largest growth rate and whether the multiphase flows could develop like the data seemed to indicate are an open question. There has also considerable effort to understand whether the reservoir of heat in the outer regions of clusters can be transferred to the center, which could effectively stabilize any initially thermally unstable parcels of gas.

Field (1965) originally discussed the origin of thermal instability due to the emission of radiation. He found that for all X-ray temperatures the gas is thermally unstable and the growth rate is fastest on the smallest scales. He further found that the small scale perturbations are damped by conduction so that the growth rates will be faster on somewhat larger scales. A number of authors (Malagoli et al. 1987; White \& Sarazin 1987a; Balbus 1988; Loewenstein 1989; Balbus \& Soker 1989) studied thermal instability in the context of gravitational field. Balbus (1991) noted that some of the thermal instability arguments are inapplicable in a cluster gravitational potential. It is possible that overdense parcels of plasma can come to equilibrium at a lower adiabat deep in the potential (Cowie et al. 1980). Kim \& Naravan (2003b) argue that the radial modes are unstable even in the presence of conduction.

Nulsen (1986) and Thomas et al. (1987), however, considered the development of perturbations into cooling flows and discussed the possible importance of magnetic fields in pinning parcels of plasma to the general hydrodynamic flow. Loewenstein (1990) discussed the importance of the magnetic field in altering the instability conditions by effectively eliminating the buoyancy problem by Balbus \& Soker (1989) with magnetic stresses. Balbus (1991) later confirmed these instabilities but stressed the importance of inefficient conduction for these conditions. However a particular, and not explained, spectrum of density perturbations is required to obtain the inferred relation $\dot{M} \propto r$ (Thomas et al. 1987; Tribble 1989). Binnev (2004) argues that multiphase flows do not occur in real clusters. 


\subsubsection{Standard Multiphase Cooling Flow Model}

The standard multiphase model simplifies the physics of the flow by assuming that radiative cooling dominates the flow and then looking at the relative amount of radiation emitted at each temperature over the cooling flow volume. This can be seen as a starting point to testing the idea that cooling flows are operating in the cores of clusters. If we restore Equation 24 to the full timederivative form and then integrate over volume and neglect any heating or additional cooling we obtain,

$$
\rho\left(\frac{5}{2} \frac{d T}{d t}-\frac{1}{\rho} \frac{d p}{d t}\right) d V=-n_{e} n_{H} \Lambda(T, Z) d V .
$$

We define a mass loss rate per time, $\dot{M}$, and a differential X-ray luminosity, $d L_{x}$, according to

$$
-\dot{M} \equiv \rho \frac{d V}{d t} ; \quad d L_{x} \equiv n_{e} n_{H} \Lambda(T, Z) d V .
$$

Then the Equation 30 simplifies to,

$$
\dot{M}\left(\frac{5}{2} d T-\frac{1}{\rho} d p\right)=d L_{x} .
$$

This can be rewritten as,

$$
\frac{d L_{x}}{d T}=\dot{M}\left(\frac{5}{2}-\frac{1}{\rho} \frac{d p}{d T}\right) .
$$

In general, $\frac{d p}{d T}$ will be set by the local gravitational field and magnetic pressure. If the gravitational field is relatively smooth as can be expected in dark matter haloes, then the pressure will remain nearly constant in small regions that will begin cooling. Whether this occurs depends on the development of thermal instability as discussed in the previous section. If $\frac{d p}{d T}$ term is small, this expression simplifies to

$$
\frac{d L_{x}}{d T_{K}}=\frac{5}{2} \frac{\dot{M} k}{\mu m_{p}}
$$

which is known as the standard isobaric cooling flow model. If the density is constant which would result from high magnetic pressure, it alternatively 
reduces to

$$
\frac{d L_{x}}{d T_{K}}=\frac{3}{2} \frac{\dot{M} k}{\mu m_{p}}
$$

which is for isochoric cooling. An adiabatic equation would result if cooling were ineffective and the gravitational potential was strong and the left hand side would be zero. In general, $\frac{d p}{d T}$ could be quite complicated, but note that it is fairly well established observationally that over the whole cooling flow volume the temperature derivative, $\frac{d T}{d r}$ is positive and the pressure derivative is negative, $\frac{d p}{d r}$. Therefore, the last term is likely to be positive, and the relative amount of X-ray luminosity would be emitted according to somewhere in between the previous two equations if X-ray radiative cooling dominated the energy release.

Note that Equation 33 is just the first law of thermodynamics $(d Q=d U+$ $\left.p d V=\frac{3}{2} N k d T+d(p V)-V d p=\frac{5}{2} N k d T-V d p=\frac{5}{2} N k d T-N \frac{d p}{\rho}\right)$ differentiated with respect to time $\left(\frac{5}{2} N k \frac{d T}{d t}-\frac{N}{\rho} \frac{d p}{d t} k T=\frac{d Q}{d t}\right)$ with $\mathrm{X}$-ray cooling as the only heat loss term.

Equation 34 is also frequently expressed in terms of the emission measure,

$$
\frac{d E M}{d T_{K}}=\frac{5}{2} \frac{\dot{M} k}{\mu m_{p}} \frac{1}{\Lambda}
$$

This then can be used in conjunction with the atomic physics necessary to produce an X-ray spectrum as shown in Figure 4, which can be compared with the data. X-ray spectroscopy has demonstrated that this model is incomplete and therefore we have most likely neglected additional heating or possibly cooling in our derivation of this model. The following section describes the instrumentation and analysis necessary to test it. 
Isobaric Multiphase Cooling-Flow Model

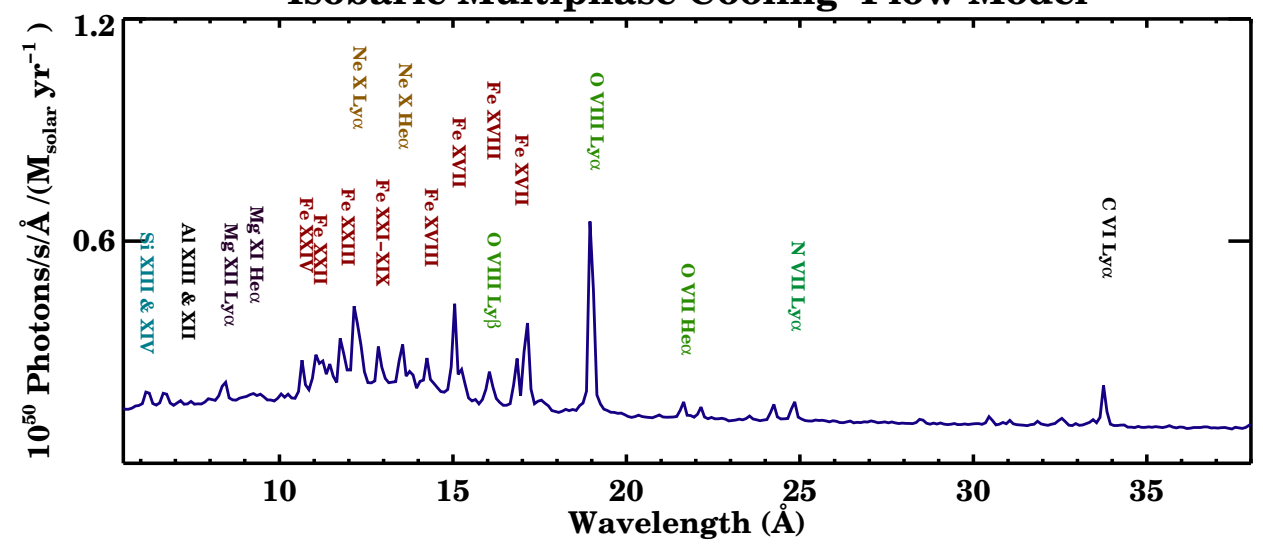

Fig. 4. The standard isobaric cooling-flow model. The model is produced by summing collisionally-ionized X-ray spectra within a temperature range such that the relative amount of luminosity per temperature interval is a constant. This model predicts relatively prominent iron L shell transitions between 10 and $17 \AA$ that arise from a wide range of temperatures. In particular, Fe XVII which is emitted between $500 \mathrm{eV}$ and $1 \mathrm{keV}$ has strong emission line blends at 15 and $17 \AA$. 


\section{X-ray Instrumentation and Observational Techniques}

A number of X-ray instruments have been launched above the atmosphere, which is opaque to X-rays, to study X-ray sources in the last 40 years. X-ray telescopes typically reflect X-rays at grazing incidence using mirros coated with a high-Z material. CCDs, proportional counters, and microchannel plates have been used to record the positions of X-ray photons and make low resolution energy measurements. Crystals, reflection and transmission gratings have been used to disperse X-rays and produce high resolution spectra.

Below, we first discuss some of the important characteristics of X-ray telescopes and the relevant instrumentation that is used to study clusters of galaxies in X-rays. Then, we discuss the analysis techniques that are used to interpret the data collected from these satellites.

\subsection{X-ray Telescopes and their Relevance to Clusters}

X-ray photons from astrophysical sources have been detected by a number of $\mathrm{X}$-ray telescopes. For observations of clusters of galaxies, several instrument characteristics have been important for gaining a complete description of the X-ray emission.

Spectral Resolution: First, the spectral resolution or resolving power $\left(\frac{\lambda}{\Delta \lambda}\right.$ or $\left.\frac{E}{\Delta E}\right)$ affects the ability to extract useful information from the spectrum. For typical temperatures in clusters of galaxies, the Fe L complex most strongly constrains the distribution of temperatures. Merely detecting or not detecting an emission line from a given charge state from a given ion, places narrow constraints on the distributions of temperatures in the plasma. The required resolution to resolve lines from individual Fe L ions is about 100 at $1 \mathrm{keV}$. Most $\mathrm{X}$-ray detectors, which historically have been either proportional counters or solid-state devices, have been unable to achieve this resolution and therefore the Fe L complex appears as an unresolved bump in the spectrum. Proportional counters work by the incoming X-ray being photoelectrically absorbed an inert gas atom followed by the measurement of the electrical discharge induced by the cascade Auger electrons and the further release of fluorescent X-rays. They typically achieve resolution near 20\%. CCD devices work by the $\mathrm{X}$-ray creating a photo-electron in the silicon, which creates a electron-hole pair cascade through ionization. The electrons drift to a set of surface contacts due to an electric field and the number of electrons is used to measure the energy of the incident X-ray. These typically have resolving powers near 10 or 20 at $1 \mathrm{keV}$. These devices can, however, measure an average temperature accurately from the shape of the continuum, which is sensitive to the 
bremsstrahlung emission, or from the centroid of the Fe L complex. These instruments, however, have difficulty contraining the distribution of temperatures composing a single spectrum.

Dispersive instruments, which either use a grating or crystal, have achieved high spectral resolution in the Fe $\mathrm{L}$ band. Crystals disperse X-rays according to the Bragg condition in which constructive interference is set up by the X-ray wavelength being equal to a multiple of the projected crystal spacing. Reflection and transmission gratings disperse X-rays by a constructive periodic surface where the X-ray wave will interfere constructively for a given angle of incidence. Both the Reflection Grating Spectrometers on XMMNewton, and the Focal Plane Crystal Spectrometer on the Einstein Observatory produced high resolution X-ray spectra of clusters. Future missions may include non-dispersive microcalorimeters, which use the temperature change of a cryogenically-cooled absorber due to the photo-electron to measure the energy of the X-ray. The Astro-E1 and Suzaku missions included a microcalorimeter, but unfortunately the rocket failed for Astro-E1 and the microcalorimeter did not operate long enough to observe cosmic X-rays for Suzaku. A microcalorimeter can achieve sufficiently high spectral resolution in the Fe K band such that few hundred $\mathrm{km} / \mathrm{s}$ velocities of the intracluster medium could be measured.

Effective Area and Exposure Time: The effective collecting area and the exposure time of the observation determine the number of photons collected. The number of photons enters into the effectiveness of the observation in a number of ways. Typically, tens of thousands of photons are collected in a given observation. Hundred of photons are necessary to make any detailed surface brightness image. Thousands of photons are necessary to make detailed spectroscopic measurements of the temperature. Tens of thousands of photons are necessary to make detailed measurements of elemental abundances as well as the construction of the differential emission measure.

Field of View: Field of view determines the fraction of photons from a cluster that are detected. Often, the field of view has been smaller than the sizes of typical nearby clusters and therefore are an important factor in comparing results from different instruments.

Spectral Bandpass: The spectral bandpass of most X-ray instruments has been quite large and for the most part encompassed most of the X-ray spectral band. Most of the photons from clusters of galaxies are emitted below $2 \mathrm{keV}$, and most instruments have had high efficiency at these energies.

Angular Resolution: Angular resolution is an important factor for spatiallyresolved spectroscopy. XMM-Newton (with FWHM of 6") and Chandra (with FWHM of 0.5 "), for example, have been able to perform spatially-resolved 
photometry on kiloparsec scales for nearby clusters.

\subsection{Analysis Techniques}

Analysis techniques play a vital role in the interpretation of X-ray spectra from clusters of galaxies. The data analysis is usually quite complex compared to the techniques that can be applied to data from unresolved sources. In addition, the instrument response functions are actually quite complex, and therefore the application of these functions is usually problematic and results often are somewhat model dependent.

X-ray photons that are recorded in detectors after being reflected and dispersed by optics have three quantities that are measured: two detector coordinates, $x$ and $y$ and one energy measurement, $p$. These are indirectly related to the position of the photon on the sky, $\phi$ and $\psi$, and the photon's intrinsic energy, $e$. For dispersive spectrometers the relationship between these three variables and their detector counterparts are very indirect and the full convolution has to be considered. For non-dispersive spectrometers, the relationship is more direct so approximations can be used. For example, it is customary to assume that $(x, y) \approx(\phi, \psi)$ when extracting a spectrum to perform spatiallyresolved spectro-photometry or to assume that $p \approx e$ to construct an image.

The detection probability, $D$, for a photon emitted from solid angle position, $\Omega$ and energy, $e$ with measured values of detector coordinates $(x, y)$ and CCD pulseheight $p$ is given by

$$
D(x, y, p)=\int d e d \Omega R(x, y, p \mid e, \Omega) \frac{d^{2} F(e, \Omega)}{d \Omega d E}
$$

where $\frac{d^{2} F}{d \Omega d E}$ is the spatially-varying spectral source model and $R$ is the instrument response function. All existing data analysis methods either solve this integral by, 1) making approximations about the response function, 2) use a Monte Carlo approach (Peterson et al. 2004), or 3) perform an integration over the solid angle and just study the spectra 4) or perform an integration over the spectra and just study the image. One can easily see some of the reasons for confusing results on spectra from clusters of galaxies. It is often somewhat ill-defined by what one analysis would mean when compared to an analysis from a different instrument without specifying the full source function, $\frac{d^{2} F}{d \Omega d E}$.

Note that most analyses construct a response matrix and an ancillary response file, which are used in spectral codes like XSPEC (Arnaud 1996) or SPEX (Kaastra 1996). These codes multiply a source spectrum on a grid by the response matrix, which relates the input spectrum to the model observed 
spectrum. The model observed spectrum can be compared to the real data by statistical tests, typically a binned $\chi^{2}$ calculation. The response matrix $\left(R_{m}\right)$ /ancillary response $(A)$ file approach is a simplification of the response function above by assuming the following separation,

$$
R(x, y, p \mid e, \phi, \psi) \sim R_{M}(p \mid e) A(e)
$$

where $A$ is constructed separately for each spectrum depending on the spatial position $(x, y)$ of the photons being studied. This approach may or may not be appropriate for a particular analysis.

The source function is in turn related to the function in Equation 7 in $§ 3.1 .5$ differentiated with respect to spatial coordinates.

$$
\frac{d^{2} F}{d \Omega d E}=\int_{0}^{\infty} d T \frac{d \alpha}{d E} \frac{d^{2} E M}{d \Omega d T}
$$

where $\frac{d^{2} E M}{d \Omega d T}$ is the differential emission measure per solid angle. This function is the true observable if the plasma is assumed to be in collisional equilibrium. Note that if a given patch of the sky is assumed to be isothermal this will directly give a measurement of the density, since the volume element can be related to the angular coordinate $\Omega$ for an assumed source distance, assuming some geometry to obtain the depth. The temperature structure in clusters is never completely isothermal, so a variety of temperatures will be sampled along the line of sight.

We will not review all work in dealing with these data analysis problems, but refer to the individual work in the following section for more details since it can vary considerably between authors. 


\section{X-ray Spectra of Cooling Clusters}

A wealth of information has been collected by a number of X-ray satellites on cooling core clusters. Recently, there have been some strict tests to various aspects of the cooling-flow scenario. We review many of the previous spectroscopic and imaging results that led to the formation of the coolingflow model for cluster thermal evolution. We then discuss how high resolution $\mathrm{X}$-ray spectroscopic results have questioned a number of assumptions in the interpretation of the previous work. These observations have largely galvanized support around the idea that the thermodynamic evolution of cluster of galaxies is considerably different than was previously thought. We then review some recent X-ray imaging and spectro-photometric work that has left some clues to the resolution of the cooling-flow problem. Finally in this section, we define the structure of the cooling-flow problem in clusters of galaxies.

\subsection{Early Work on Imaging Observations}

The cooling time of the ICM is less than the Hubble time at the centers of many clustes as demonstrated by Lea et al. (1973) with the Uhuru mission. Imaging observations established the existence of sharp surface brightness peaks in some cluster of galaxies. These surface brightness peaks and short cooling times were interpreted as strong evidence for the existence of cooling flows. The existence of sharp surface brightness peaks were found by the Copernicus satellite (Fabian et al. 1974; Mitchell et al. 1975), rocket missions (Gorenstein et al. 1977), and SAS-3 (Helmken et al. 1978). Later missions established the widespread nature of the phenomenon by establishing that approximately $50 \%$ of all clusters contained sharp surface brightness profiles. The Einstein Observatory (Stewart et al. 1984; Arnaud 1998), EXOSAT Observatory (Lahav et al. 1989; Edge \& Stewart 1991; Edge et al. 1992), and ROSAT Observatories (Allen et al. 2001a) confirmed this picture with observations of hundreds of nearby clusters of galaxies.

Considerable work was done to establish the implied mass deposition rates from the cooling flows (Jones \& Forman 1984). The mass deposition rates, $\dot{M}$, were estimated to be between 1-10 solar mass per year for a giant elliptical galaxy and 100-1000 solar masses per year for the largest clusters of galaxies. A first order estimate of the mass deposition rate is simply made by taking the total gas mass calculated from the density profile and dividing that by the cooling time at the edge of the region. Alternatively, this can be written as the luminosity of the cooling flow volume divided by the temperature (in units of $\mathrm{kT}$ ) times the mean mass per particle. More detailed calculations take into account the work done on the gas by its compression in the gravitational 
potential (Thomas et al. 1987; White \& Sarazin 1987a, b), 1988; Allen et al. 1993). Generally, this effect is thought to reduce the overall level of mass cooling by at most a factor of two from the amount predicted without gravitational compression (Arnaud 1998).

\subsection{Early Work on Low Resolution Spectroscopy}

The modeling of the X-ray spectra of cooling clusters has become considerably more mature in 30 years. In addition, some of the early work with coolingflow models has now been discounted. This work exemplifies the difficulty in applying models at low spectral resolution. We briefly discuss some of the major changes to modeling the X-ray spectrum of cooling clusters.

The Areil- $\mathrm{V}$ satellite established that the emission from clusters of galaxies was thermal (i.e. likely in collisional equilibrium) by detecting the emission lines from the Fe K $\alpha$ blend (Fe XXV He $\alpha$, Fe XXVI Ly $\alpha$ ) from the Perseus cluster (Mitchell et al. 1976). Previously, there were arguments for the origin of the X-ray emission from clusters as either inverse compton scattering of microwave background photons off relativistic electrons or emission from a collection of unresolved X-ray binaries. The detection of thermal emission, however, established that the hot gas was trapped in the dark matter potential and emitted at a temperature close to what was expected from the velocity distribution of member galaxies.

After the thermal model was established, hundreds, if not thousands, of clusters had their "mean" temperature measured with solid state detectors or proportional counters most frequently on the Einstein, EXOSAT, BeppoSAX, and ASCA observatories. This work established relationships between many observables in clusters. In particular, relationships between temperature and luminosity (David et al. 1993; White et al. 1997; Markevitch 1998; Wu et al. 1999; Horner et al. 1999), mass (Nevalianen et al. 2000; Xu et al. 2001), and entropy (Ponman et al. 1999) indicate that mean cluster gas properties roughly follow from what is expected from self-similar gravitational collapse. There may be some deviation from these relations, more preferentially in lower mass clusters indicating the importance of non-gravitational processes in cluster formation and evolution.

It was discovered quite early that many clusters do not have an isothermal structure (Ulmer \& Jernigan 1978) and have softer X-ray spectra in the core. This implies lower mean temperatures. A number of more complicated spectral models were later used to represent the X-ray emission from cooling-flows with EXOSAT, Einstein, Beppo-SAX, and ASCA observations. Two temperature models were used by a number of authors to represent the distribution of tem- 


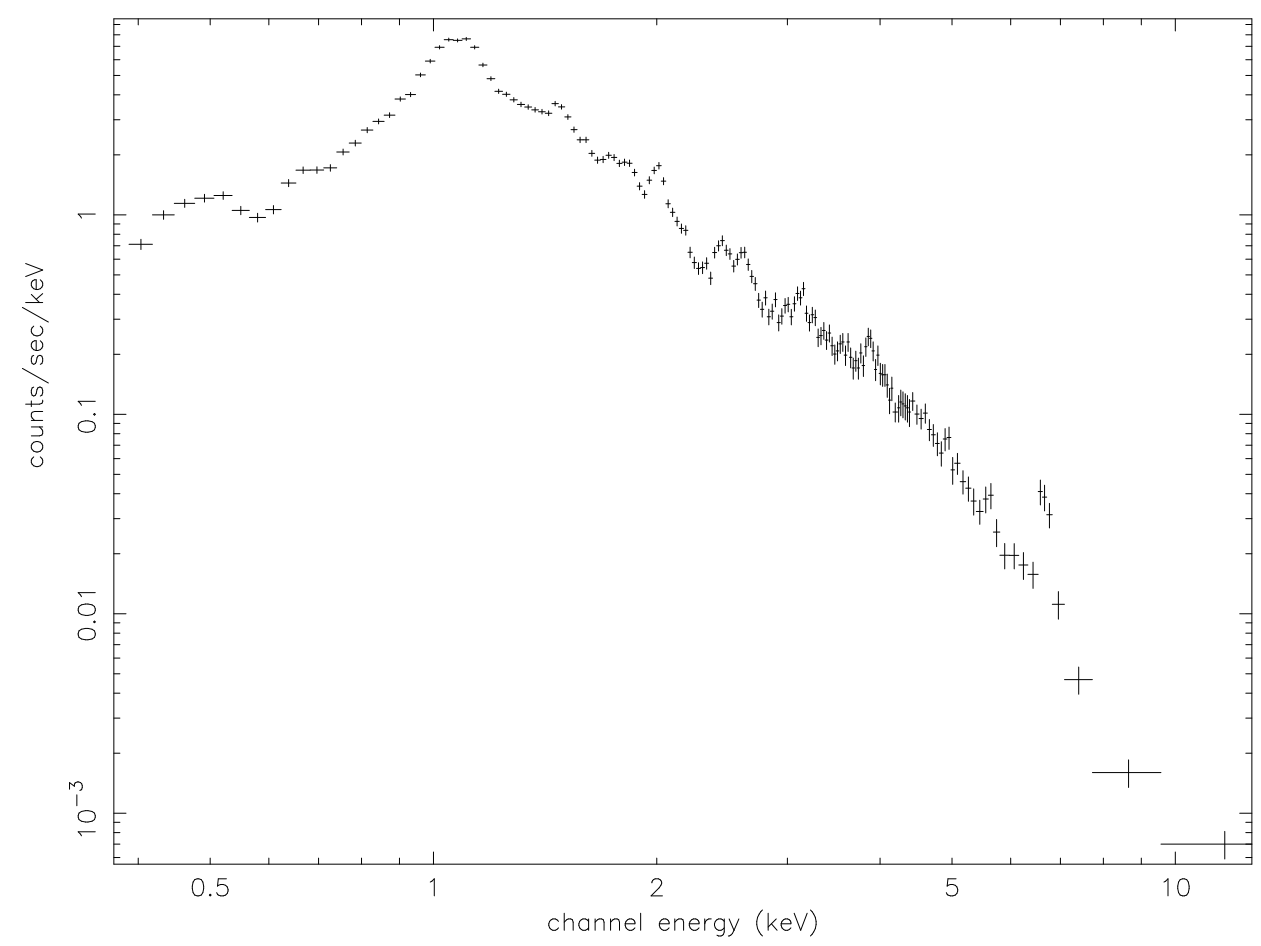

Fig. 5. The X-ray spectrum of the central region of the Virgo cluster of galaxies obtained with the ASCA observatory. This spectrum is one of the highest quality spectra obtained prior to the launch of the RGS instruments on XMM-Newton. The bumps in the spectrum are due to unresolved blends of emission lines. In particular, the bump near $1 \mathrm{keV}$ is due to a forest of Fe $\mathrm{L}$ shell transitions, which are quite sensitive to the temperature of the plasma.

peratures in the cores of clusters (Matsumoto 1996; Ikebe et al. 1997; Xu et al. 1998; Fukazawa et al. 1998, 2000; Makishima 2001). An alternative interpretation of the cold gas in the cores of cluster was developed by Makishima (2001) where the dark matter halo of the $\mathrm{cD}$ galaxy is responsible for an unmixed ICM component.

Others used the cooling flow model discussed in $§ 3.3$ modified by an absorber. The absorber was necessary to reduce the soft X-ray emission at low energies observed by White et al. (1991). In retrospect it is clear that these observations were demonstrating a lack of emission at low energies relative to the cooling flow model, although this was not explicitly stated. In particular, the relatively low spectral resolution made it difficult to distinguish between models.

The absorber in these models was modelled typically as a single absorbing screen (White et al. 1991; Johnstone et al. 1992; Fabian et al. 1994; Buote \& Fabian 1998; Buote et al. 1999, 2000a; Allen et al. 2000, 2001a). The screen was placed between the cluster and the observer, but the assumption was that this absorption was representing cold gas in the center of the cluster. The absorption typically had column densities near $10^{21} \mathrm{~cm}^{-2}$, which would have implied large quantities of cold molecular gas if it was distributed uniformly. Initial attempts 
to find this quantity of gas were unsuccessful (O'Dea et al. 1998), although considerable cold material has since been found by Edge et al. (2001) and Salome \& Combes (2004). Another model used was a cooling flow model with a single absorption edge. The absorption edge had an energy near $0.7 \mathrm{keV}$, which could have been from ionized Oxygen possibly from warm $\left(10^{6} \mathrm{~K}\right)$ material (Buote et al. 2000b) or dust (Allen et al. 2001b). Later, high resolution Reflection Grating Spectrometer (RGS) observations demonstrated that the single edge model mimicked the effect of lack of emission from colder Fe ions. It is interesting to note, in particular, that the fitted energy of the single absorption edge was slightly lower than the $3 \mathrm{~s}-2 \mathrm{p}$ transitions of Fe XVII, and would therefore optimally appear to absorb cooler emission from the standard cooling flow model.

Many observations generally established the cooling time is a fraction of a Gyr in relaxed clusters. They also clearly indicated that the temperature was lower where the central surface brightness was highest. These two fact are now shown clearly with recent data from the Chandra observatory in Figure 6 and 7 .

\subsection{Focal Plane Crystal Spectrometer}

The first high resolution observations of cooling clusters comes from the Focal Plane Crystal Spectrometer (FPCS) on the Einstein observatory. This instrument used a crystal to disperse X-rays according to the Bragg condition. It therefore could produce a high resolution spectrum by scanning in angle and therefore scanning in wavelength. This worked for an extended source like a cluster as well.

Unfortunately, there were only a limited number of these observations and the count rates were not particularly high. These observations demonstrated the existence of line emission from 8 ions (Canizares et al. 1979, 1982) in four different clusters. Some of these observations are contradicted by Reflection Grating Spectrometer observations as will be described in the next section, so it is likely that these observations were partially compromised by high background rates and, in some cases, relatively few photon counts.

\subsection{Reflection Grating Spectrometer Observations}

Observations made at high spectral resolution with the Reflection Grating Spectrometers on XMM-Newton have greatly clarified the observational interpretation of the soft X-ray spectra of cooling clusters. Although some information can be gained from the exact shape of the Fe L complex with low spectral 


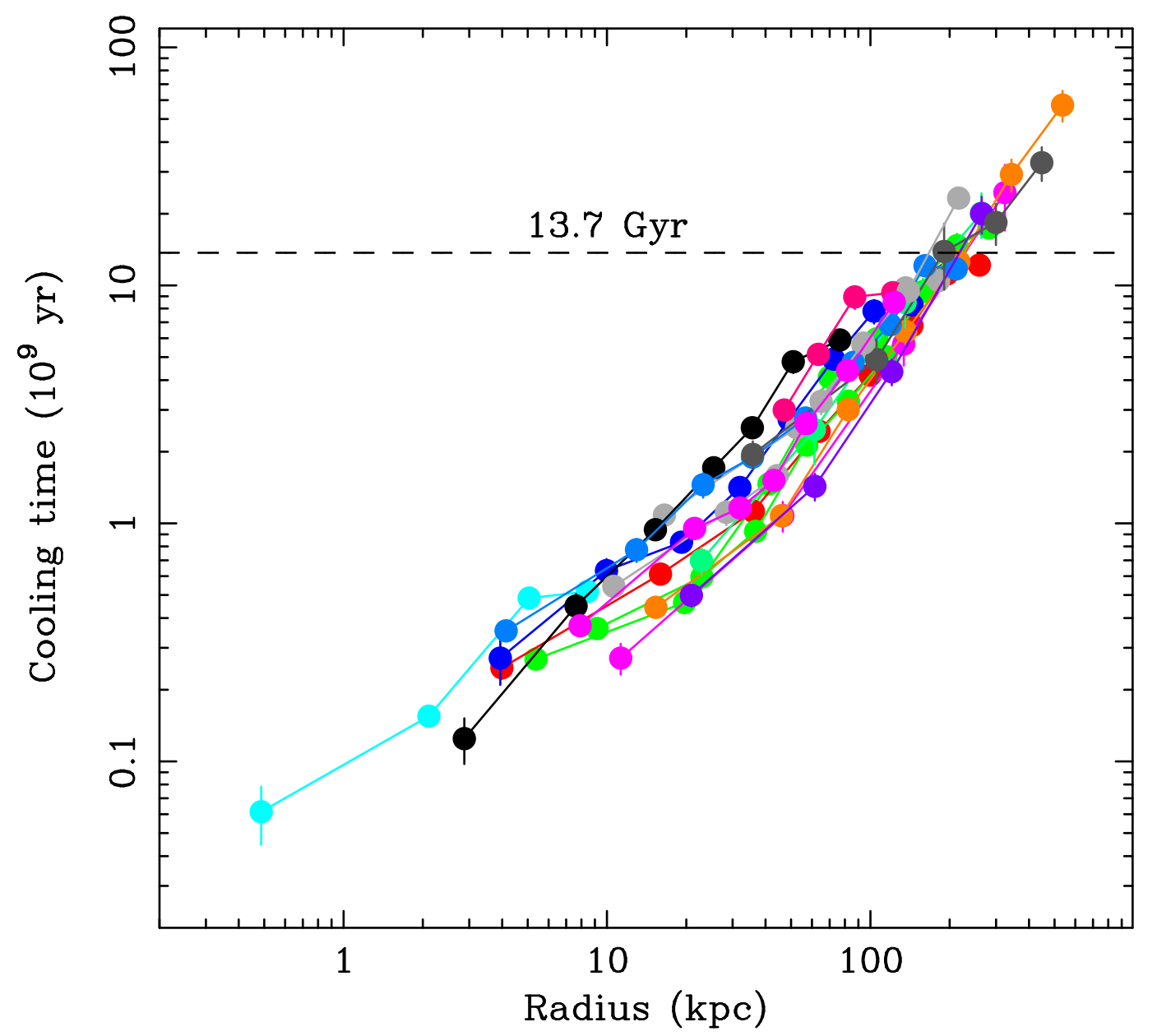

Fig. 6. The cooling time as a function of radius for a sample of relaxed clusters as measured with Chandra. If left undisturbed, all plasma within 100 to $200 \mathrm{kpc}$ would have sufficient time to cool. Figure adapted from Voigt \& Fabian (2004).

resolution spectrometers, RGS observations resolve emission lines from each Fe L charge state (Fe XXIV through Fe XVII) and therefore can place crucial constraints of the amount of gas present between temperatures of 0.4 and 4 $\mathrm{keV}$.

The initial application of the standard cooling flow model to a high resolution X-ray spectrum is shown in Figure 8 (Peterson et al. 2001). The top panel shows the spectral prediction between 5 and $35 \AA$. Emission lines are expected from Fe L ions in roughly equal strength between 10 and 18. Particularly prominent are emission lines from Fe XVII and 15 and 17 Angstroms. These emission lines are produced primarily from plasma between 300 and $700 \mathrm{eV}$ and represent the last major emitting ion before a cooling plasma would cool to sub-X-ray temperatures.

High resolution spectroscopic evidence for lack of cool gas has been documented in a number of clusters (Peterson et al. 2001; Tamura et al. 2001a; Kaastra et al. 2001; Sakelliou et al. 2002; Tamura et al. 2001b). Characteristi- 


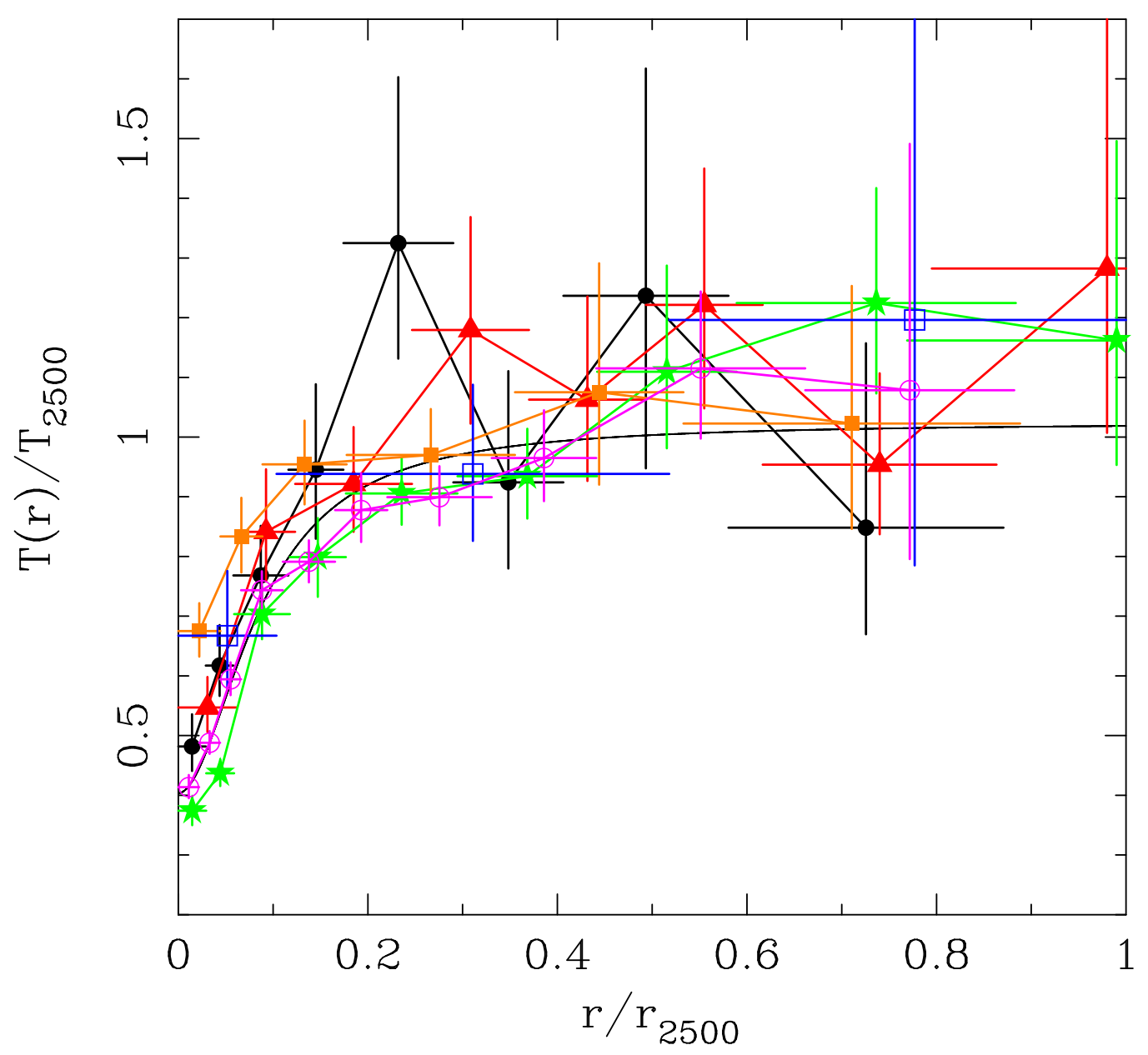

Fig. 7. The average temperature in radial bins for a sample of relaxed clusters of galaxies as measured with Chandra. The temperature and radius are scaled to $r_{2500}$. A typical cluster shows a clear decline in the average temperature at the center, which is in agreement with many spectroscopic studies over the previous two decades. Figure is adapted from (Allen et al. 2001c).

cally, gas appears to be missing at near a third of the maximum temperature. This phenomena has also been documented in elliptical galaxies. $\mathrm{Xu}$ et al. (2002) found that Fe XVII and Fe XVIII was present in the nearby elliptical galaxy, NGC 4636. O VII, however, was not detected and has not been detected in any galaxy, group, or cluster core.

A more detailed analysis of the nature of the cooling flow problem is found in Peterson et al. (2003). A sample of 14 clusters were analyzed in a uniform way to demonstrate that the cooling flow problem manifests itself at a fraction of the maximum temperature in the center. In this sample it is shown that hot clusters (5-10 keV) generally only show evidence of Fe XXIV-XXII emission and no other Fe L charge states. Intermediate temperature clusters (2-5 keV) show evidence for Fe XXIV-Fe XIX emission, but no Fe XVII and Fe XVIII. The coolest clusters and groups (less than $2 \mathrm{keV}$ ) show evidence of the entire Fe 
L series but anomalously low levels of Fe XVII emission when compared with the standard cooling flow model. All of these observations are in contradiction with the standard cooling flow model, and suggest qualitatively that the model is violated at characteristically a fraction of the temperature and is more inconsistent with the model at lower temperatures.

In this quantitative analysis (Peterson et al. 2003), the cooling flow model was separated into four different temperature ranges as shown in Figure 9. Then the normalization of each temperature range was adjusted to empirically fit the spectrum shown in Figures 10, 11, and 12. The results from this study are shown in Figures 13 and 14. These graphs plot each of the four luminosity detections for each clusters. The lines connect the points that are actual spectral detections and not just upper limits. The standard cooling flow model would be if all the points would lie on a horizontal line. A comparison of Figure 13 and 14 suggests that when plotting the differential luminosity vs. the fraction of the temperature as in Figure 14, that the trend is more systematic. Therefore, the emission of a cooling flow is consistent with a power law in differential luminosity with exponent between 1 and 2. The cooling flow model, however, is strongly ruled out in many systems.

At this point, there has been no significant evidence for any significant difference between cooling flows of similar cluster mass. This poses some challenges for heating models where there is a time-dependence to the process. There also has been no evidence from X-ray spectroscopy that there are significant quantities of plasma at low X-ray temperatures above the expectation of the cooling flow model. In other words, Figure 14 shows a monotonically decreasing violation of the cooling flow model and no evidence for gas piling up at some intermediate temperature. This presents some challenges for continuous heating model that do not reheat the gas completely. For both of these reasons, it is not straight-forward to interpret these results in the context of any heating model regardless of the mechanism.

Finally, it is worth noting that these results generally are displaying the $\mathrm{X}$ ray spectrum of the entire cooling flow volume. There is considerable work that is continuing to actually study the changes in the emission spectrum as a function of spatial position inside the cooling flow. These studies are difficult since determining a spatially-resolve differential emission measure require both high angular resolution and high spectral resolution. Following, we discuss some attempts to study the spatial-dependence of the X-ray emission of cooling flow at moderate spectral resolution. 


\subsection{Recent Spatially-resolved Spectro-photometric Observations}

The RGS results obtained at high spectral resolution have been augmented with further studies at low resolution that attempt to study the spatial distribution in greater detail (David et al. 2001; Böhringer et al. 2001; Molendi \& Pizzolato 2001; Schmidt et al. 2001; Ettori et al. 2002; Johnstone et al. 2001). In general, it has also been demonstrated that the low spectral resolution observations obtained with XMM-EPIC and Chandra, which are far more numerous, are generally consistent with the RGS observations. Significantly smaller mass deposition rates have been measured when the cooling flow model has been applied. Similarly, cut-off cooling flow models have been shown to be consistent with the data. Needless to say, a larger range of models can be applied to the low resolution data and still be statistically consistent with the data. In general, it appears that there is no strong evidence for any significant amount of cold gas (below $\frac{1}{3}$ of the maximum temperature) in any cluster.

The radial profile of the temperature distribution in cooling flows is currently in dispute. It is clear that the ICM has a much narrower range of temperatures than compared to the standard cooling flow model as established by the RGS observations. It is not so clear, however, how narrow that distribution is at

any given radius. Molendi \& Pizzolato (2001) have argued that the ICM is nearly isothermal at a given radius and any departure from this is due solely to azimuthal variations. Buote et al. (2003) and Kaastra et al. (2004) have argued, however, that nearly a factor of two range of gas temperatures exists at any radius.

X-ray cavities have been discovered in a number of X-ray imaging observations. The cavities are presumably excavated by cosmic rays produced in outbursts by the central radio source (McNamara et al. 2001). These cavities are important in the study of cooling flows for a number of reasons. First, they represent direct empirical evidence that the cores of cluster have been disturbed. The work done by expansion of the bubbles assuming all the energy is deposited in the cooling flow is within a factor a few to the value required (Birzan et al. 2004). Second, they are relatively intact and coherent structures indicating the level of suppression of thermal transport processes possibly by a magnetic field. Therefore, they provide a source of external energy to the ICM and detailed study will eventually give us a complete picture of the transport of that energy.

Constant pressure surface brightness discontinuities, or "cold fronts", are present in many clusters of galaxies (Markevitch 2000). Cold fronts show that there are vast quantities of plasma oscillating with respect to the gravitational potential. Some may be cooler, denser groups that have fallen to the center, whereas others may be cooled gas that already was present in the core of the cluster 
but is oscillating because of a merger in the cluster outskirts (Churazov et al. 2003).

Fine ripples have been identified in the cluster gas of Perseus (Fabian et al. 2003a). These features are extremely weak and more study is needed to measure the temperatures across the discontinuities and confirm their properties. Detections of these features have also been claimed in Hydra A (Nulsen et al. 2004) and M 87 (Forman et al. 2003). It is unclear if these are an entirely separate phenomena from cold fronts. Given their proximity to the center, Fabian et al. (2003a) suggested that these were weak shocks and sound waves. In addition, Sanders et al. (2004) have identified an asymmetric "swirl" in the temperature distribution in the Perseus cluster. This observational feature is may be due to the effect of off-center cluster mergers.

\subsection{Observations of Cataclysmic Variables}

Although not the subject of this review, it is certainly worth noting that the $\mathrm{X}$-ray spectrum of some binary accreting white dwarf systems, cataclysmic variables, actually resembles the X-ray spectrum of the standard cooling flow model (Mukai et al. 2003). The flow behind the standing shock in an accreting white dwarf involves the same physical processes as those expected for a cooling flow in a cluster although the geometry may be different. Although there are a great number of differences between clusters of galaxies and cataclysmic variables, these observations can be used to confidently say there is no mistake in applying a model for a radiative-cooling dominated plasma. Furthermore, this points to no major difficulties in the atomic physics models or subtle problem with the plasma physics arguments we have used.

\subsection{Definition of the Cooling Flow Problem}

We now briefly discuss what we believe the cooling flow problem is, and how it might be resolved. Clearly, the problem is quite complex and it is difficult not to see the problem in historical terms. Peterson et al. (2003), for example, discussed a difference between the soft X-ray cooling-flow problem and the mass sink cooling-flow problem. The former refers to the recent discrepancy seen in the soft X-ray spectrum between what was predicted and what was observed. The latter refers to the difficulty in detecting any by-products in cooling clusters from the hypothesized cooling-flow plasma.

These definitions, however, might just categorize our ignorance of the solution to the problem. The major difficulty is that: 1) the cluster plasma loses energy by emitting the very X-rays we detect, 2) efficient and distributed heat sources 
are difficult to construct, 3) the cluster plasma appears to cool most of the way, but 4) evidence for complete cooling is utterly lacking. The cooling-flow problem as we see it is to understand what happens in the middle of that process. After examining whether cooling flows are ruled out, we discuss many ideas that might alleviate the cooling-flow problem.

Note, from Fig. 6, that time variability on intervals longer than $10^{8}$ yr cannot be the solution. 

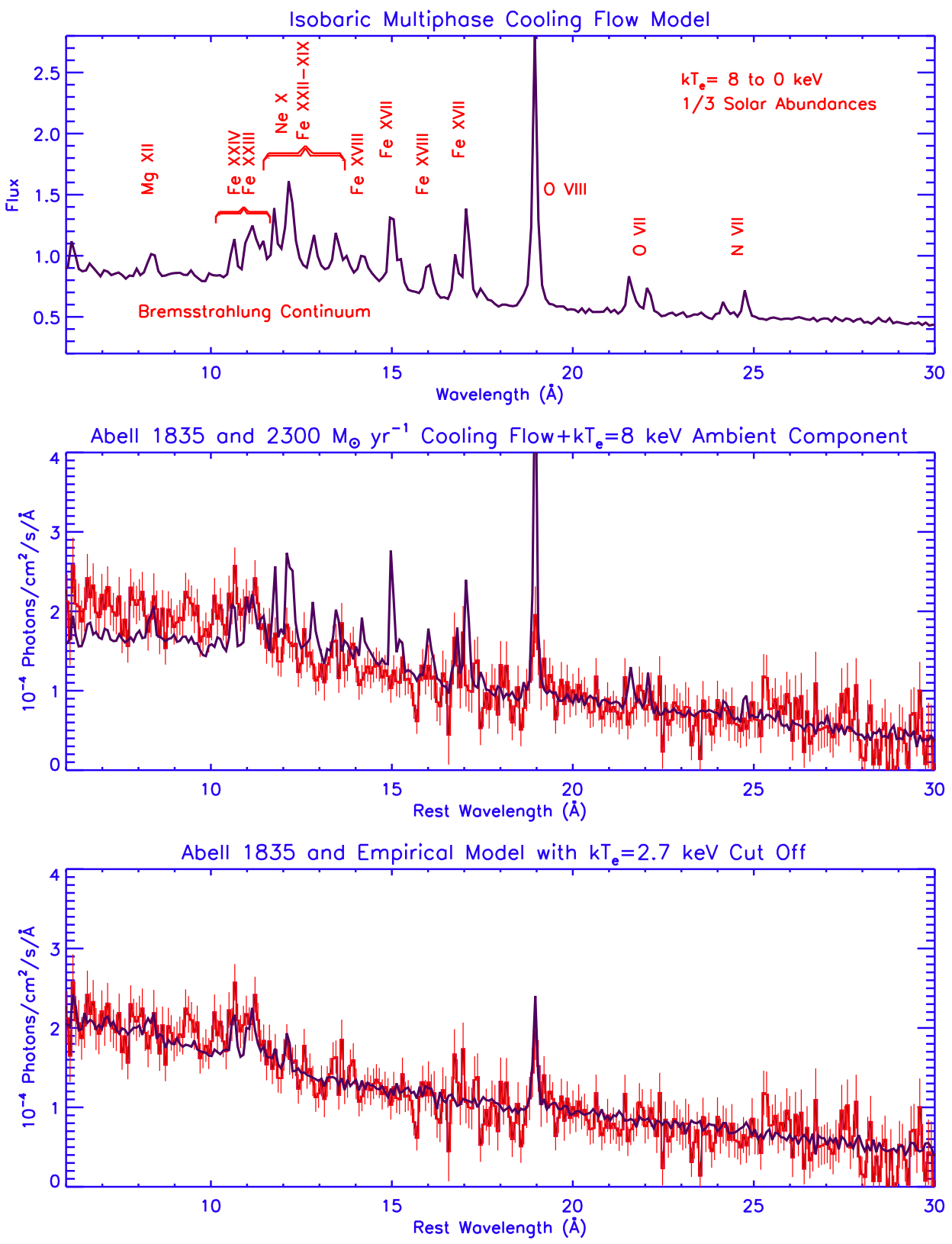

Fig. 8. Three panels showing the cooling-flow model applied to the X-ray spectrum of the putative massive cooling-flow, Abell 1835. The top panel shows the standard isobaric cooling flow model (see the previous figure). The middle panel shows the model (blue) and the data obtained from the Reflection Grating Spectrometer on XMM-Newton (red). The model is clearly inconsistent with the X-ray spectrum, particularly in the prediction of Fe XVII emission line blends at 15 and $17 \AA$. The bottom panel shows the cooling flow model compared with the data, except all emission coming from temperatures below $2.7 \mathrm{keV}$ is suppressed. The explanation for the success of this model is not known. Adapted from Peterson et al. (2001). The spectrum is taken from a 5 by 20 arcminute region of the core. 


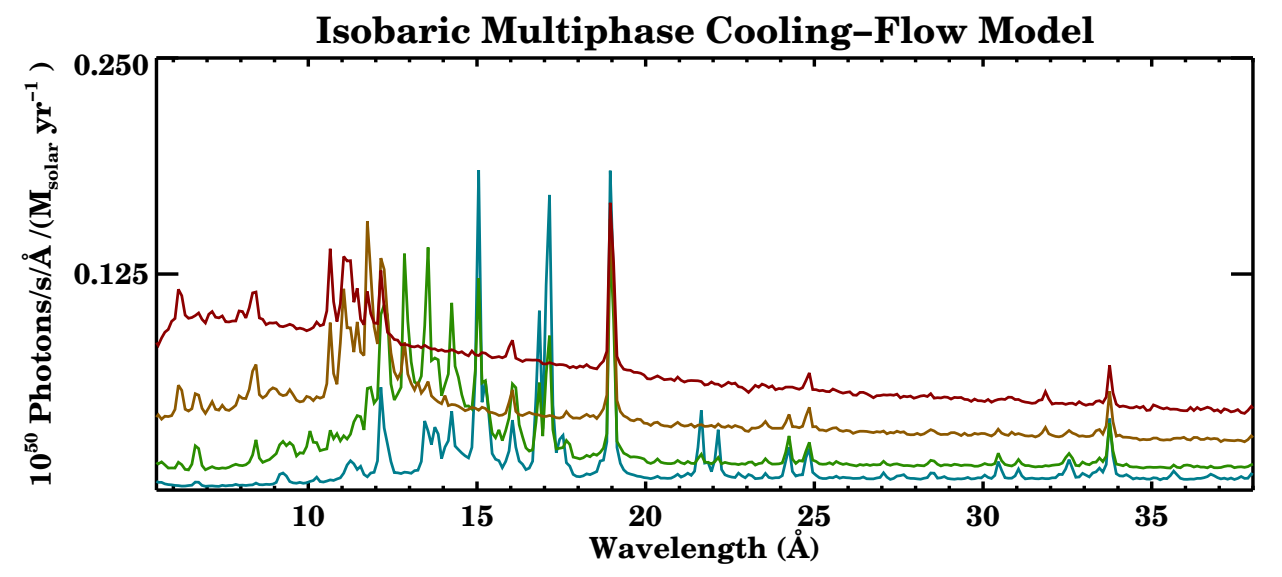

Fig. 9. The isobaric cooling flow model divided into the contributions from various temperature ranges, 0.375 to $0.75 \mathrm{keV}$ (blue), 0.75 to $1.5 \mathrm{keV}$ (green), 1.5 to $3 \mathrm{keV}$ (yellow), 3 to $6 \mathrm{keV}$ (red). 


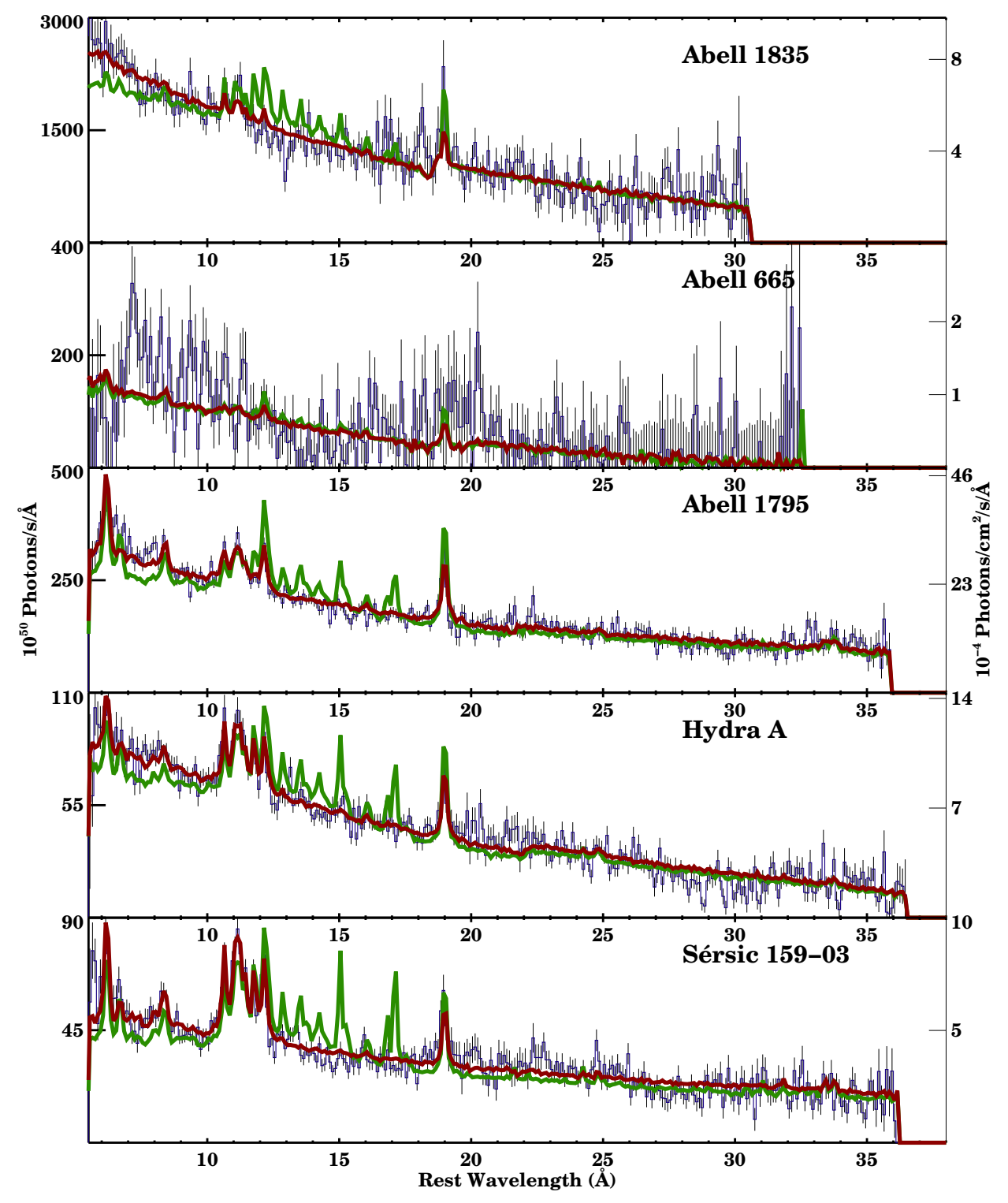

Fig. 10. Two models compared with the Reflection Grating Spectrometer data of a sub-sample of massive cooling flows. The blue histogram is the RGS data, the green curve is the standard cooling-flow model, and the red is an empirical model where emission from the standard cooling-flow model is allowed to be adjusted in specific temperature ranges. The spectrum is taken from a 5 by 20 arcminute region of the core. 


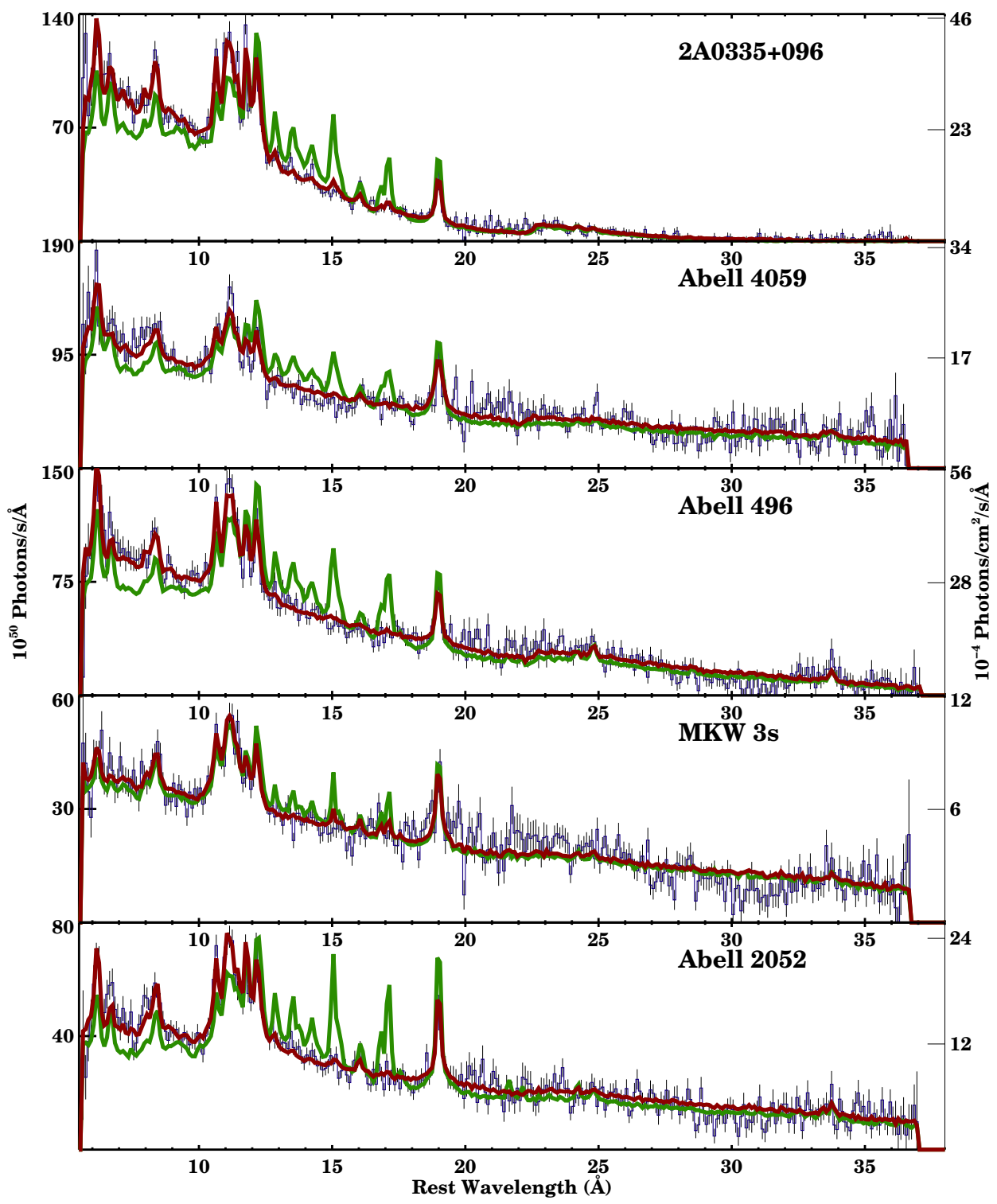

Fig. 11. The same as the previous figure, but for a sample of intermediate mass clusters. 


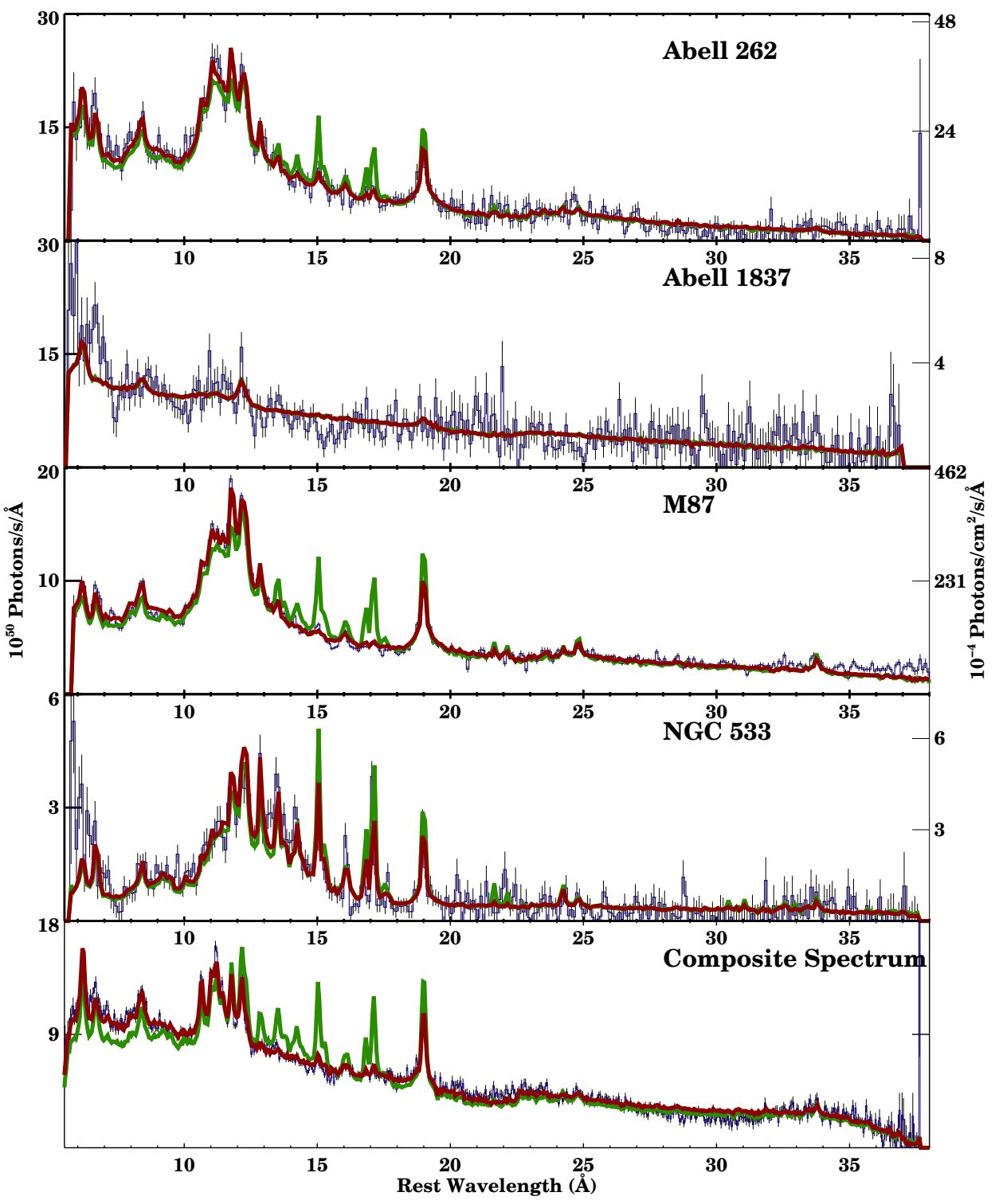

Fig. 12. The same as the previous two figures, but a sample of low mass clusters and groups of galaxies. 


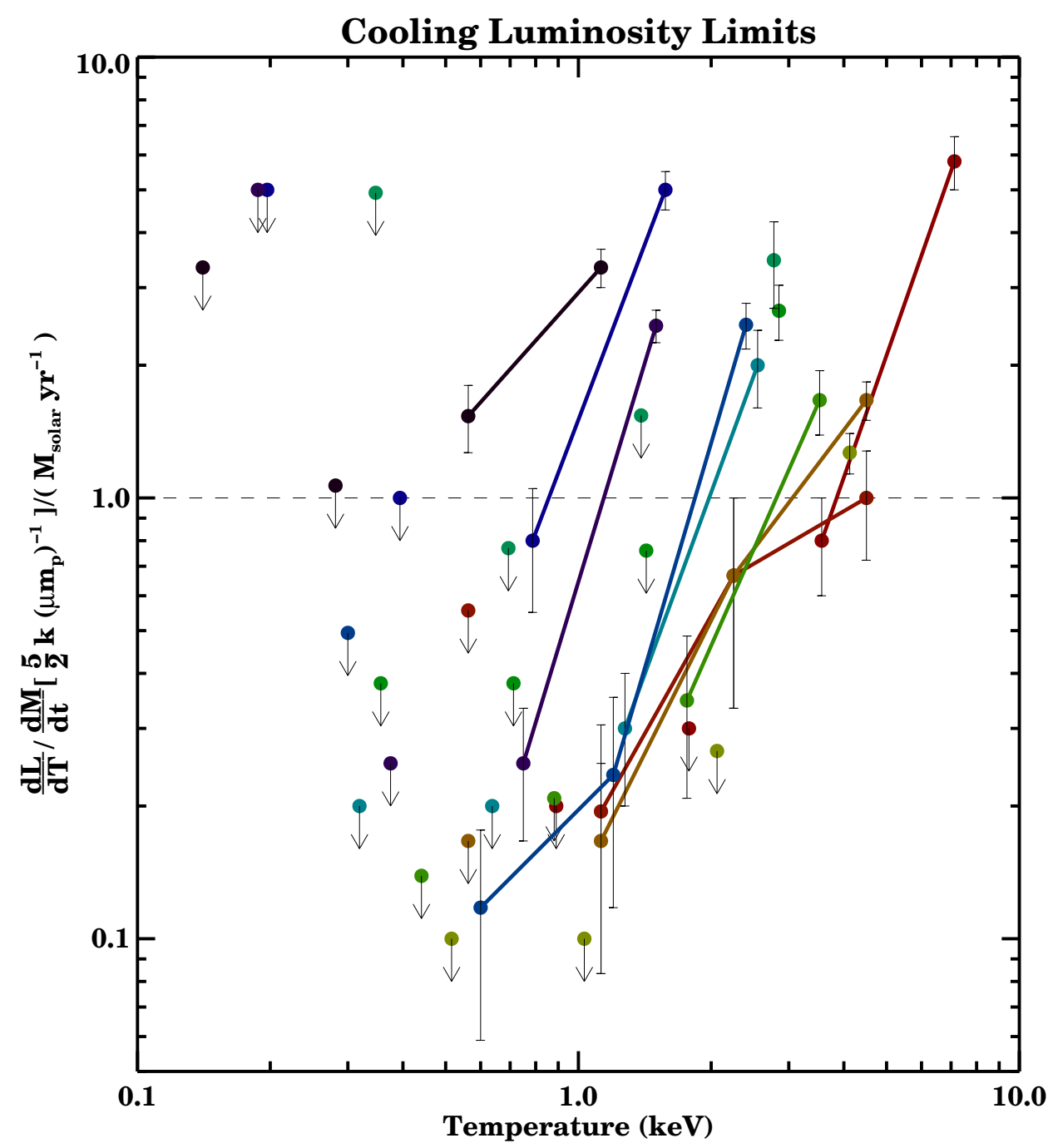

Fig. 13. The relative amount of emission in various temperature ranges from a sample of 14 clusters obtained with the Reflection Grating Spectrometer on XMM-Newton. Each cluster is a different color, and each cluster has four points. Points that are not upper limits are connected by a straight line. The standard cooling flow model predicts the same amount of emission in each temperature range or a horizontal line. The data, however, are clearly inconsistent with that model. The detected emission has a much steeper distribution in temperature and many upper limits are a factor of several below the prediction. Adapted from Peterson et al. (2003). 


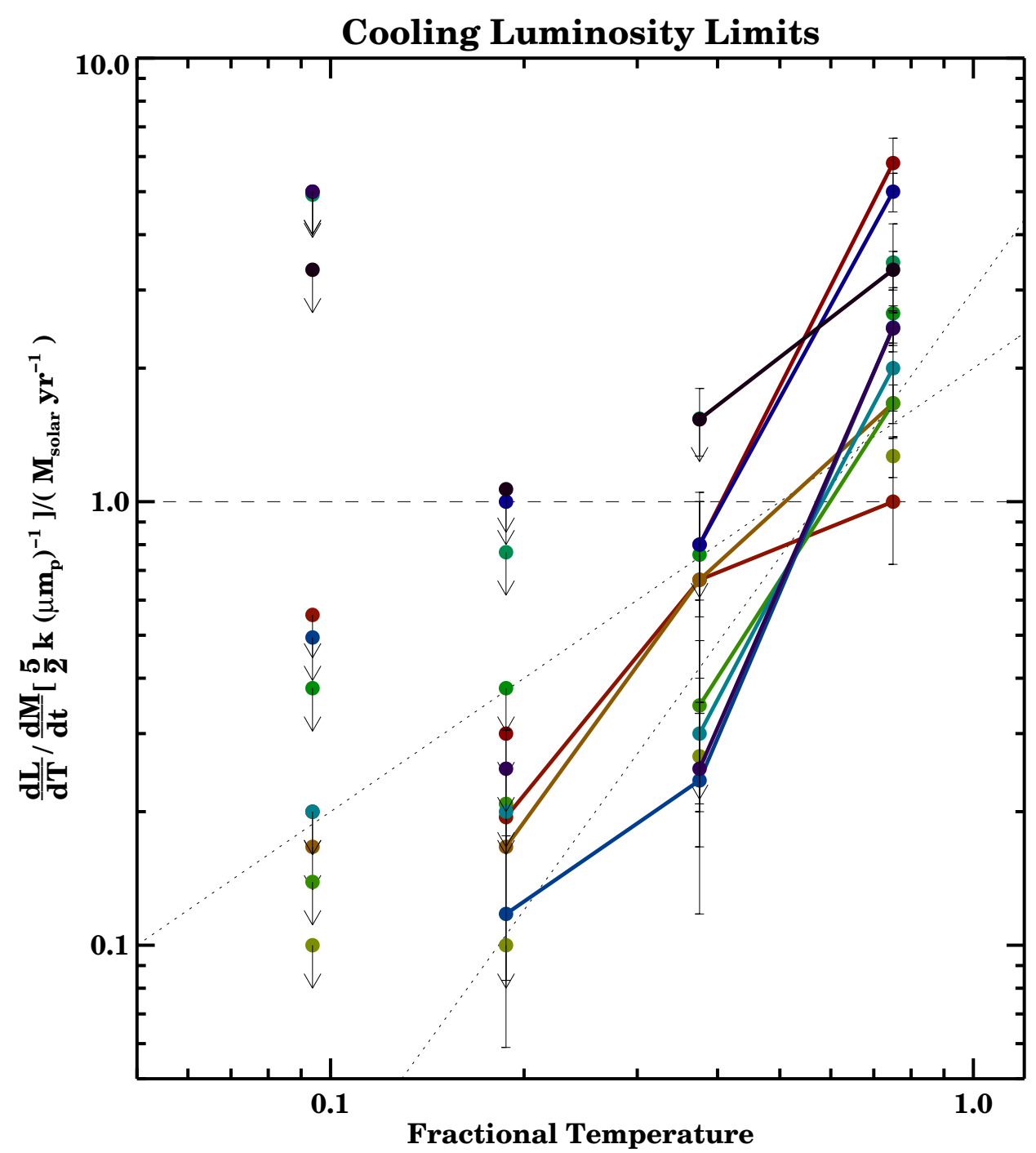

Fig. 14. Same as the previous figure, except the points are normalized to the maximum temperature in the fit. A more systematic trend is seen where the emission follows a linear or quadratic form in temperature (dotted lines). There is significant scatter in the points, but there does appear to be a "self-similar" violation to the standard cooling flow model (dashed line). Adapted from Peterson et al. (2003). 


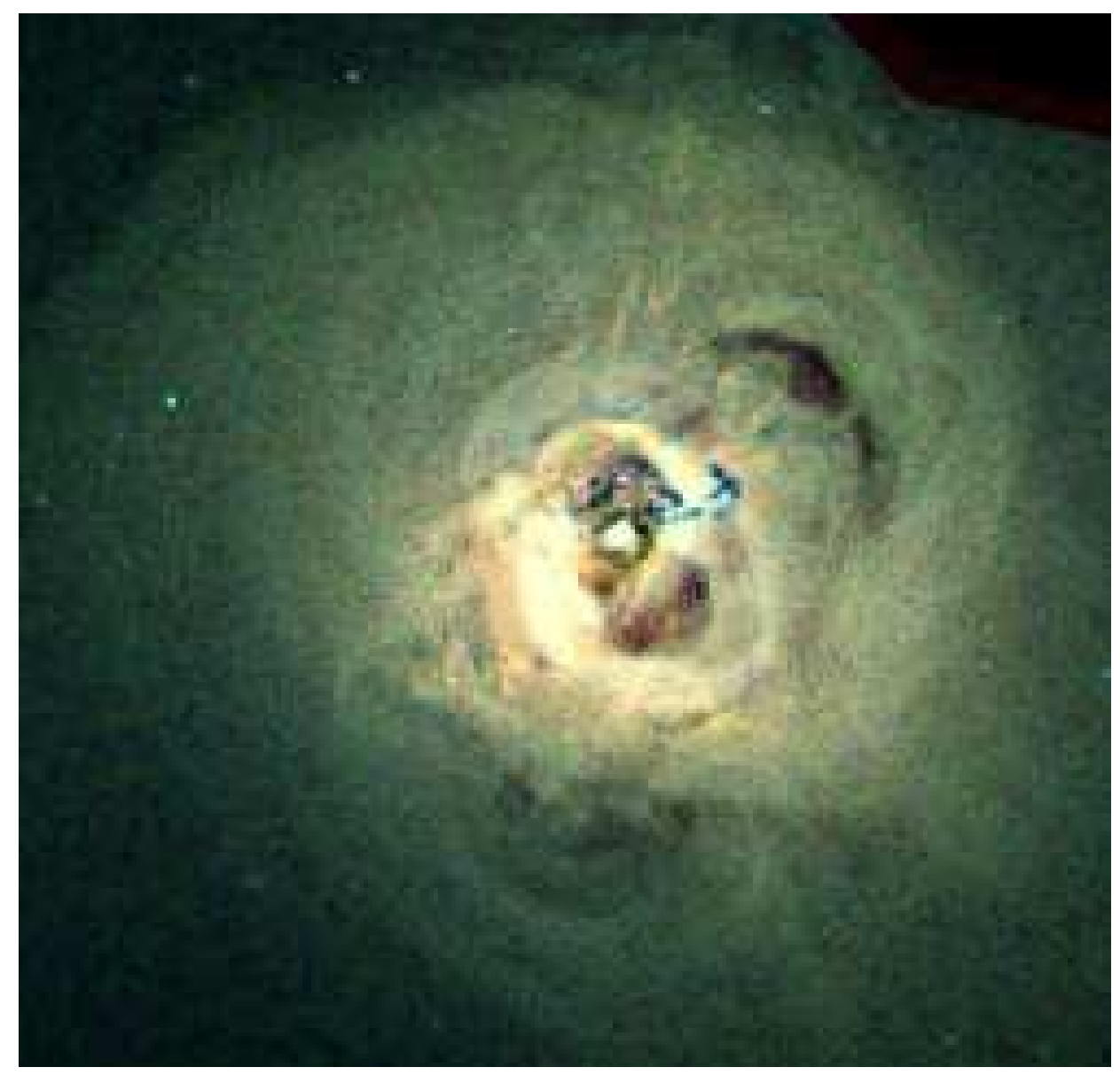

Fig. 15. High resolution X-ray image of the Perseus cluster of galaxies. The bright source in the center is the active galactic nucleus of NGC 1275. The two adjacent holes in the X-ray emission as well as the mushroom shaped depression in the upper right are believed to be cavities in the ICM that have been excavated by cosmic rays expelled from the active galactic nucleus. Figure is adapted from Fabian et al. $(2005 b)$. 


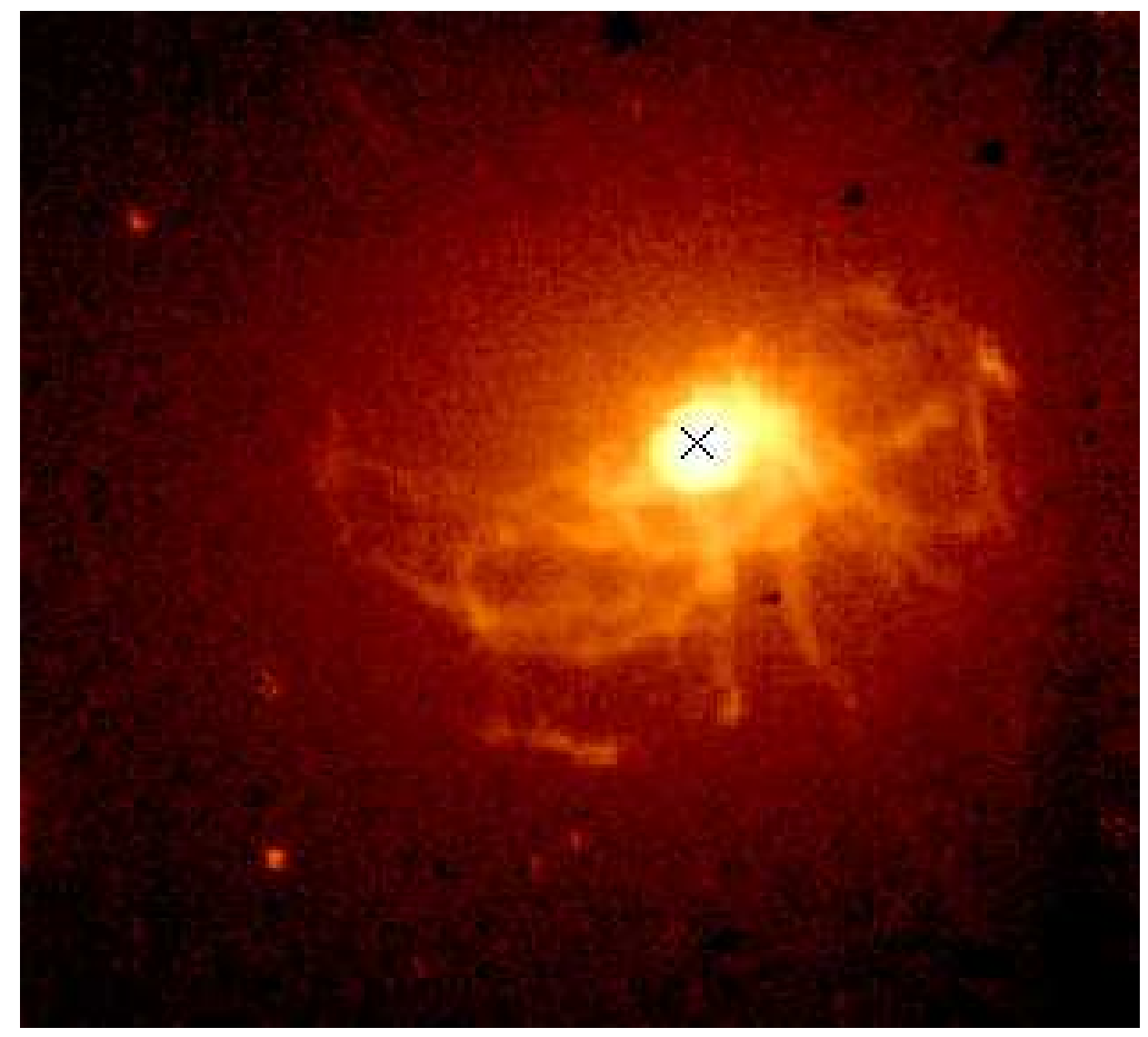

Fig. 16. Image of the Centaurus cluster of galaxies overlayed with $\mathrm{H} \alpha$ emission showing a filamentary structure. Figure is adapted from Crawford et al. (2005). 


\section{$6 \quad$ Are cooling flows ruled out?}

In summary, problems with the simple cooling flow picture emerged from two fronts. The first is the enormous implied mass deposition in some objects where for example $\dot{M} \sim 1000 \mathrm{M}_{\odot} \mathrm{yr}^{-1}$ (e.g. PKS 0745-191; Fabian et al. 1985) which is then not seen in cooled form (the 'mass sink' problem). The central galaxy of this, and many other brightest cluster galaxies where the surrounding gas have $t_{\text {cool }}<3$ Gyr, has much excess blue light, optical/UV/IR emission-line nebulosity (Crawford et al. 1999 and refs. therein) and even molecular gas (Edge et al. 2001). The total star formation rate and gas mass is often one to two orders of magnitude below such a large value of $\dot{M}$ operating for several Gyr. The flow could be somewhat intermittent but not overly so or short central cooling times would not be so common (Peres et al. 1998). A nonstandard IMF for the stars is one possibility (Fabian et al. 1982), but has to be extreme (Prestwich et al. 1997).

The second problem is that a simple cooling flow spectrum is a poor fit to the data in the soft X-ray band (White et al. 1991; Johnstone et al. 1992; Fabian et al. 1984). What was usually done was to fit the model spectrum of an isobaric cooling flow (Johnstone et al. 1992) plus an isothermal spectrum to represent the luminosity from gravitational work done, to the data from the inner regions. A deficit in soft X-ray emission was found in the data when compared with the model. This was then modelled as photoelectric absorption due to an absorber intrinsic to the central regions. The lack of any obvious absorption in very soft ROSAT spectra was attributed to emission from the intervening gas in the cluster (Allen \& Fabian 1997).

Photoelectric absorption intrinsic to the cluster does not however appear in the spectra of the iet in M87 (Böhringer et al. 2002) or the nucleus of NGC 1275 (Churazov et al. 2003). Nor is it apparent in the detailed XMM/RGS spectra of cool cluster cores (Peterson et al. 2001; Tamura et al. 2001a; Kaastra et al. 2001; Sakelliou et al. 2002; Peterson et al. 2003). Absorption has generally been abandoned as an explanation, although no detailed test has been made of a model in which the absorption is intimately linked with the coolest cooling gas clouds. Note though that the presence of warm emission-line nebulosities, dust-lanes and clear optical absorption lines (Carter et al. 1997; Sparks et al. 1997) show that at some level there must be distributed intrinsic absorption.

Curiously, the 'missing' soft X-ray luminosity (i.e. the above soft X-ray deficit) is close to the luminosity emitted from the emission-line nebulosity commonly found in these systems (Fabian et al. 2002a; Soker et al. 2004). This has led to the suggestion that the gas is cooling but not radiatively once its temperature has dropped to $\sim 1 \mathrm{keV}$ or so. It can be cooled by mixing (Fabian et al. 2002a) or conduction (Soker et al. 2004) with cold gas. A possible picture would be 
that the gas cools from 100 to within $20 \mathrm{kpc}$ radiatively as a single-phase flow by which radius its temperature has dropped by a few. It then shares its temperature with cooler gas and thereby rapidly drops down to below $10^{6} \mathrm{~K}$. The cooler gas is close to the peak of the cosmic cooling curve and radiates the energy in the UV and optical bands (and IR if there is molecular gas and dust). Such a solution to the soft X-ray deficit still suffers from the mass sink problem.

A further situation which could give a soft X-ray deficit is if the metals in the ICM are highly inhomogeneous. They are presumably introduced in a very localized manner and at high abundance by stars and supernovae. If they do not mix but after time just reside at a similar temperature to their surroundings then when bremsstrahlung cooling dominates (which it does above about $1 \mathrm{keV}$ for Solar abundances) then all the gas cools together. But when it has cooled so that line cooling dominates, the highly enriched clumps would then cool rapidly and drop out (Fabian et al. 2001; Morris \& Fabian 2003). This has been considered in part by Böhringer et al. (2002), although no conclusive test has been performed.

The main reason that complete cooling flows, in which gas cools from the cluster virial temperature to well below $10^{6} \mathrm{~K}$, are often now considered to be ruled out is the high spectral resolution XMM/RGS data (Peterson et al. 2001; Tamura et al. 2001a; Kaastra et al. 2001; Sakelliou et al. 2002; Peterson et al. 2003). Chandra spectra lead to a similar conclusion (David et al. 2001; Allen et al. 2001a). These spectra have been covered in depth earlier in this review. We summarize here to note that most data are consistent with the ICM temperature dropping within the cooling radius by a factor of about 3 (sometimes significantly larger values are found e.g. A2597 (Morris \& Fabian 2005) and Centaurus (Fabian et al. 2005a). Data from all wavelengths are consistent with a residual flow ranging from a few $\mathrm{M}_{\odot} \mathrm{yr}^{-1}$ to atypical value of tens $\mathrm{M}_{\odot} \mathrm{yr}^{-1}$ up to $\sim 100 \mathrm{M}_{\odot} \mathrm{yr}^{-1}$ in some cases (e.g. Baver-Kim et al. (2002); Wise et al. (2004); McNamara et al. (2004)). These values are generally compatible with the ongoing star formation seen.

\subsection{Cooled gas and star formation}

The brightest galaxy in X-ray peaked clusters often has excess blue light indicating massive star formation and IR/optical/UV emission lines (Crawford et al. 1999; Donahue et al. 2000; Hicks \& Mushotzky 2005). The regions are often dusty so the determination of the star formation rate depends on uncertin dust corrections. It can be tens $\mathrm{M}_{\odot} \mathrm{yr}^{-1}$ to about $100 \mathrm{M}_{\odot} \mathrm{yr}^{-1}$ in the case of A1835. In a typical BCG it is one to ten per cent of the mass cooling rate inferred from the hotter X-ray emitting gas assuming it cools completely. 
UV-excess BCGs probably have the highest rate of star formation of earlytype galaxies at the present epoch. The brightest galaxy in non-X-ray-peaked clusters do not have this emission.

The excitation of the emission-line nebulosity, which often has a filamentary structure (e.g. the Perseus cluster: Conselice et al. 2000: the Centaurus cluster: Crawford et al. 2005; the Virgo cluster: Sparks et al. 2004) is likely related to the star formation but in detail there are problems. Hotter emission is required than expected from the stars observed and there are no stars obvious within the outer filaments. The gas, even in the outer filaments, contains dust and molecular hydrogen (Hatch et al 2005a; Jaffe et al. 2005). Simple models for the molecular gas imply higher pressures than for the surrounding gas and very short radiative cooling times (Jaffe et al. 2001; Wilman et al. 2002). The filaments may have been pulled out from a central reservoir of cold and warm gas by the action of radio bubbles (Fabian et al. 2003b; Hatch et al 2005b). Where the central gas comes from is not clear although radiative cooling is likely. A residual cooling flow is therefore taking place in many clusters.

Gas at intermediate temperatures of about $10^{5.5} \mathrm{~K}$ is seen through OVI emission with FUSE. Oegerle et al. (2001) found such emission from A2597 and recently it has been found in A426 and A1795 (Bregman et al. 2005). The inferred cooling rates in the 30 arcsec FUSE aperture are $30-50 \mathrm{M}_{\odot} \mathrm{yr}^{-1}$. 


\section{Heating}

Some heating is always expected in the central regions of clusters. Examples are supernovae (Silk et al. 1986; Domainko et al. 2004), an active central nucleus (Bailev 1982; Tucker \& Rosnen 1983; Pedlar et al. 1990; Tabor \& Binnev 1993; Binnev \& Tabor 1995) and many more recent papers cited in Sec 7.27.5), conduction (Takahara \& Takahara 1979; Binnev \& Cowie 1981; Stewart et al. 1984; Friaca 1986; Bertschinger \& Meiksin 1986; Rosner \& Tucker 1989) and many more recent papers cited in Sec 7.1).

A problem with heating the gas is that the cooling rate is proportional to the density squared whereas most heating processes are proportional to volume. This tends to make the gas unstable and means generally that the cooler denser gas will carry on cooling while hotter surrounding gas heats up. The gas appears to cool by about a factor of three and then stop cooling. A mechanism to do that is not obvious, since the gas does not appear to be piling up at the lower temperature. Indeed it seems that the gas temperature profile is "frozen" and has been so for some Gyrs (Bauer et al. 2005).

The profile of $t_{\text {cool }}$ is similar in many clusters with a common central minimum value for $t_{\text {cool }}$ of about $200 \mathrm{Myr}$ (Fig. 6). This strongly suggests that heating is continuous, at least on timescales of $10^{8} \mathrm{yr}$ or more and is spatially distributed. Moreover, no shock waves are apparent in these regions so any mechanical energy injection must be subsonic.

Brighenti \& Mathews (2003) (see also Ruszkowski \& Begelman 2002) provide several 1D simulations of a cluster core in which various levels of heat are injected at various radii. The results which best fit the temperature profile

of a real cluster are those without heating. This demonstrates that a heating model is not entirely straight-forward.

Some authors use a combination of processes. Although several are operating in any cluster, it is unlikely that they have similar weight, especially when it is recalled that the cooling flow problem exists in objects ranging from elliptical galaxies, through groups to the most massive clusters. It applies to a range of about 100,000 in X-ray luminosity and over 15 in temperature. We are therefore seeking a wide ranging, quasi-continuous, gentle, distributed heating process

We now consider conduction and AGN heating in detail before discussing other models. 


\subsection{Heat conduction}

The outer cluster atmosphere beyond the cooling radius represents a vast reservoir of thermal energy. Conduction can transfer heat into the cooler central regions. The rate of conduction is however highly uncertain due to the presence of magnetic fields which are probably tangled.

Most workers have adopted Spitzer (Spitzer et al. 1962) conductivity $\left(\kappa_{\mathrm{S}} \propto\right.$ $T^{5 / 2}$, which is appropriate for an unmagnetized gas, and then assumed a suppression factor $f$. Naravan \& Medvedev (2001) suggest that $f$ is about $1 / 5$ since field lines wander away from each other exponentially when tangled (see Tribble 1989 for another discussion).

Comparison with the data initially looked promising (Zakamska \& Naravan 2003; Voigt et al. 2002) then under further inspection ruled it out (Kim \& Naravan 2003a; Voigt \& Fabian 2004). Voigt et al. (2002) (see also Kaastra et al. 2004 and Voigt \& Fabian 2004) used the density and temperature profiles to deduce the heat transfer rate within the gas and thus the effective conductivity $\kappa_{\text {eff }}$ required. For several high temperature clusters, $\kappa_{\text {eff }}<\kappa_{\mathrm{S}}$, but for clusters where most of the gas is below $5 \mathrm{keV}$ then $\kappa_{\text {eff }}>\kappa_{\mathrm{S}}$. Then seems unlikely, however, but this may be possible (Cho \& Lazarian 2004).

Cho et al. (2003) proposed that turbulent conduction might work, whereby large subsonic eddies cause heat to be transferred in radius. This does appear to operate fast enough to explain the data (Kim \& Naravan 2003a; Voigt \& Fabian 2004) provided the gas is highly turbulent. We shall return to the issue of turbulence later when discussing heating by the central radio source $(\S 7.2)$.

We note that conduction as a solution has a fine tuning problem (Nulsen et al. 1982; Bregman 1988). If the gas starts out isothermal, then either the gas cools in which conduction cannot stop it (conduction has a steep positive temperature dependence) or conduction does work and there is no cool gas. How a profile resembling a cooling flow would occur is difficult to understand.

\subsection{Heating by a central radio source}

Most central cluster galaxies where $t_{\text {cool }}$ in the surrounding gas is less than a few Gyr have a nucleus radio source (Burns 1990). The radio luminosity of these sources has a wide range from the powerful FR II objects Cygnus A and 3C295 to weaker FR I objects such as M87. ROSAT HRI data clearly showed that the FR I lobes of 3C84, the central radio source in the Perseus cluster, have displaced the ICM and so are strongly interacting there (Böhringer et al. 


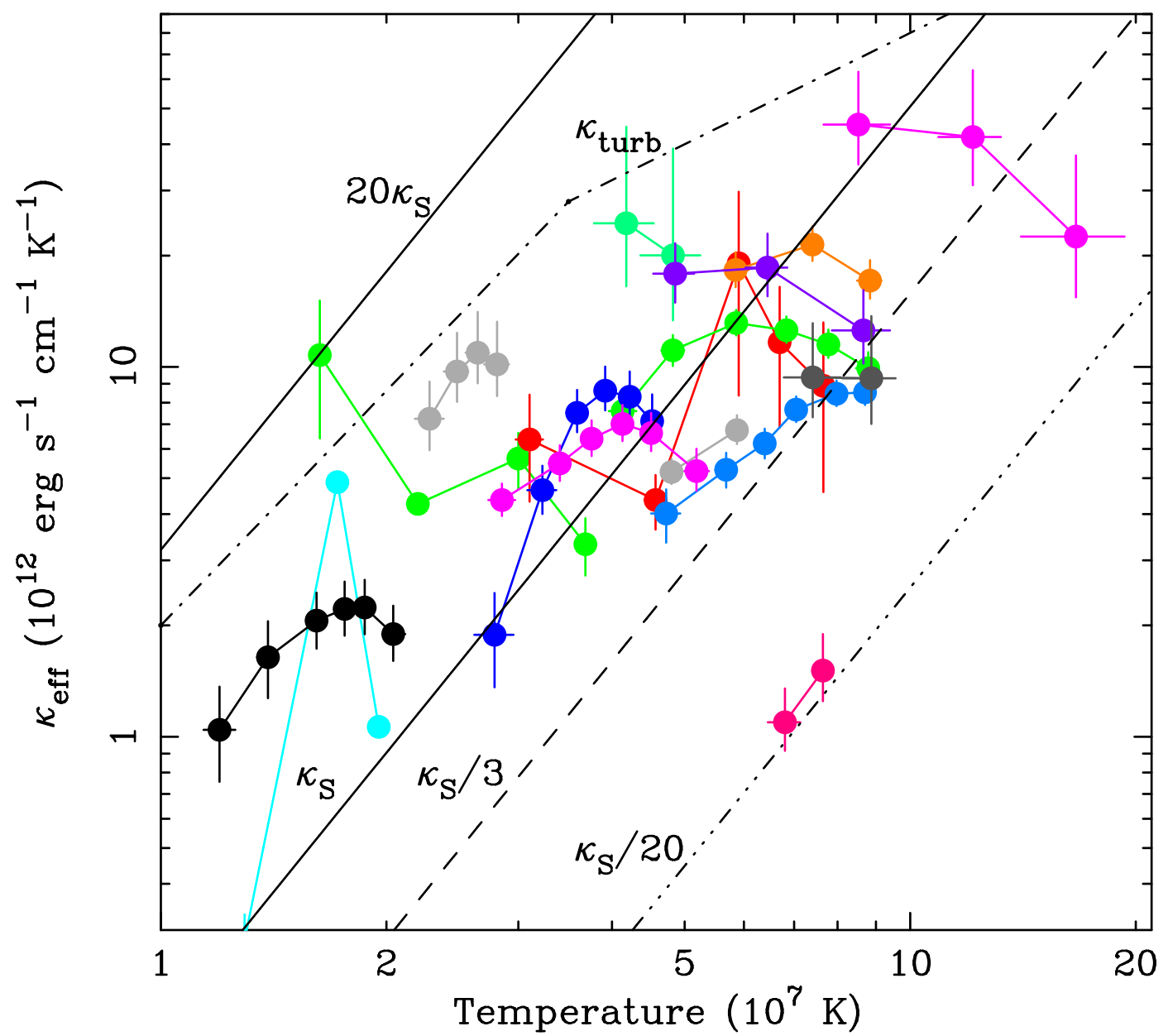

Fig. 17. The coefficient of electron thermal conduction that would be required to balance X-ray cooling in the cores of a sample of clusters of galaxies. These data are derived by measuring a mean temperature and density as a function of radius and calculating the conduction coefficient to cancel radiative cooling. Each color is a different cluster and each point is a measurement at various radii. The lines are prediction for the magnetic field free conduction coefficient of Spitzer et al. (1962). It is unclear what to expect for the effective conductivity in a cluster of galaxies with a complex magnetic field topology (see text), but it would probably be below the Spitzer value. These results probably indicate the conduction is not strong enough to cancel cooling by itself, but the value required curiously happens to be within an order of magnitude of the canonical value.

1993). The higher spatial resolution of Chandra has revealed further details of that source (Fabian et al. 2000, 2003a, 2005b) and enabled the discovery of many more, similar, interactions. 


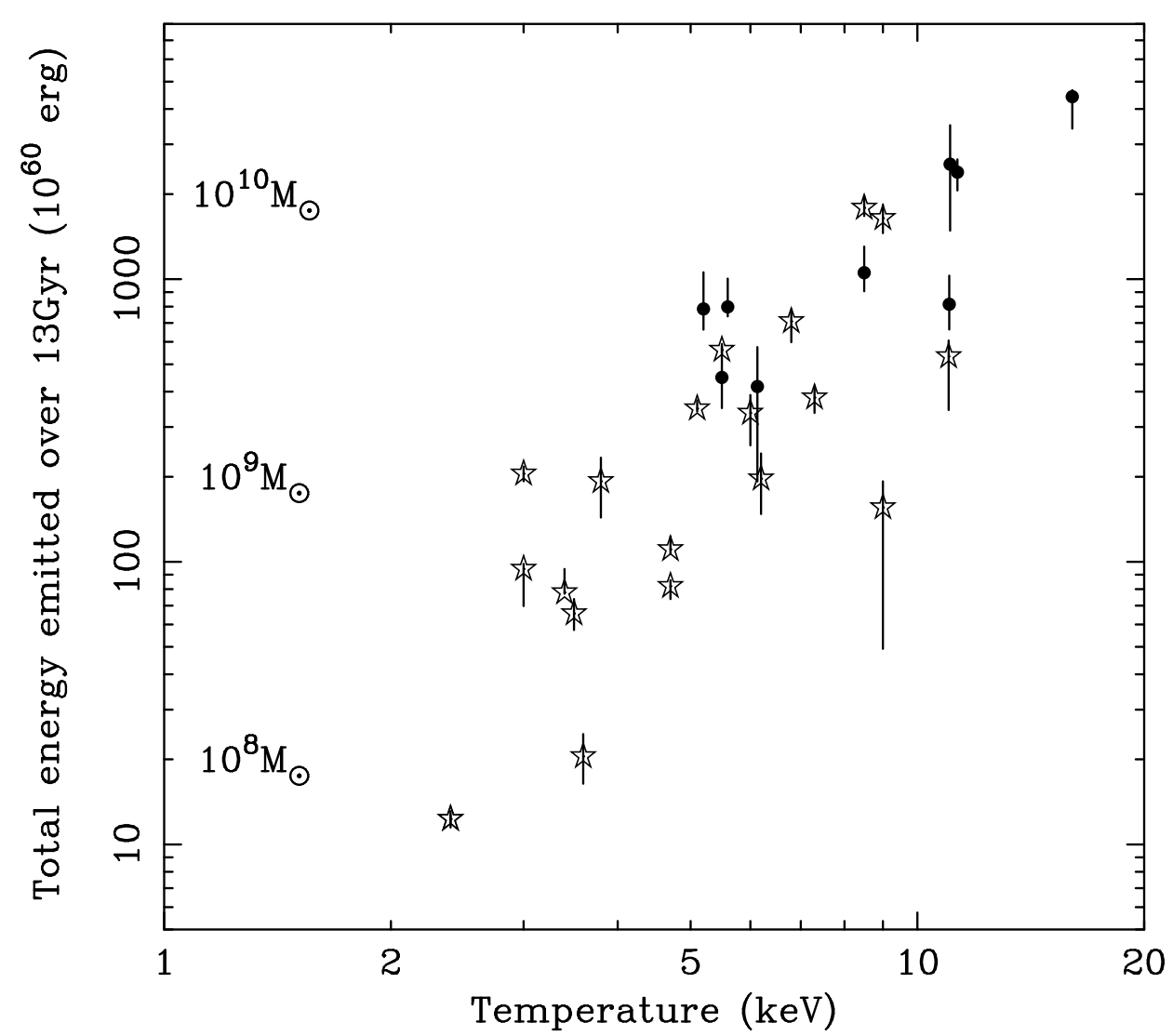

Fig. 18. The total heating power required to balance radiative cooling a sample of cooling-flow clusters over a Hubble time. The left hand side also indicates the mass of the black hole required if ten percent of its rest mass were applied directly into heating the ICM. Figure adapted from Fabian et al. (2002).

\subsubsection{Overview of radio bubbles}

Holes in the X-ray surface brightness coincident with radio lobes are commonly seen and generally referred to as radio bubbles. They are presumed to be relatively empty of normal thermal gas (see limits by Schmidt et al Schmidt et al. 2001) and mostly filled with a plasma consisting of relativistic electrons, protons and magnetic field. Striking examples apart from in the Perseus cluster are seen in Hydra A (McNamara et al. 2000); A2052 (Blanton et al. 2001); Centaurus (Fabian et al. 2000); M87 (Young et al. 2002; Forman et al. 2003);

A2597 (McNamara et al. 2001; Clarke et al. 2005) and many more (Birzan et al. 2004; Dunn \& Fabian 2004; Dunn et al. 2005).

Sometimes outer bubbles are seen (the NW one in the Perseus cluster was first seen in images from the Einstein Observatory, (Fabian et al. 1981; Branduardi-Ravmont et al. 1981) which are considered to be ghost lobes. Synchrotron and other losses have depleted the population of radio-emitting electrons to the extent that they are not detectable in the radio (particularly not at high frequencies) yet the other electrons, protons and magnetic field in the cavities still exclude the $\mathrm{X}$-ray emitting thermal gas. Weak, low-frequency radio emission pointing at 


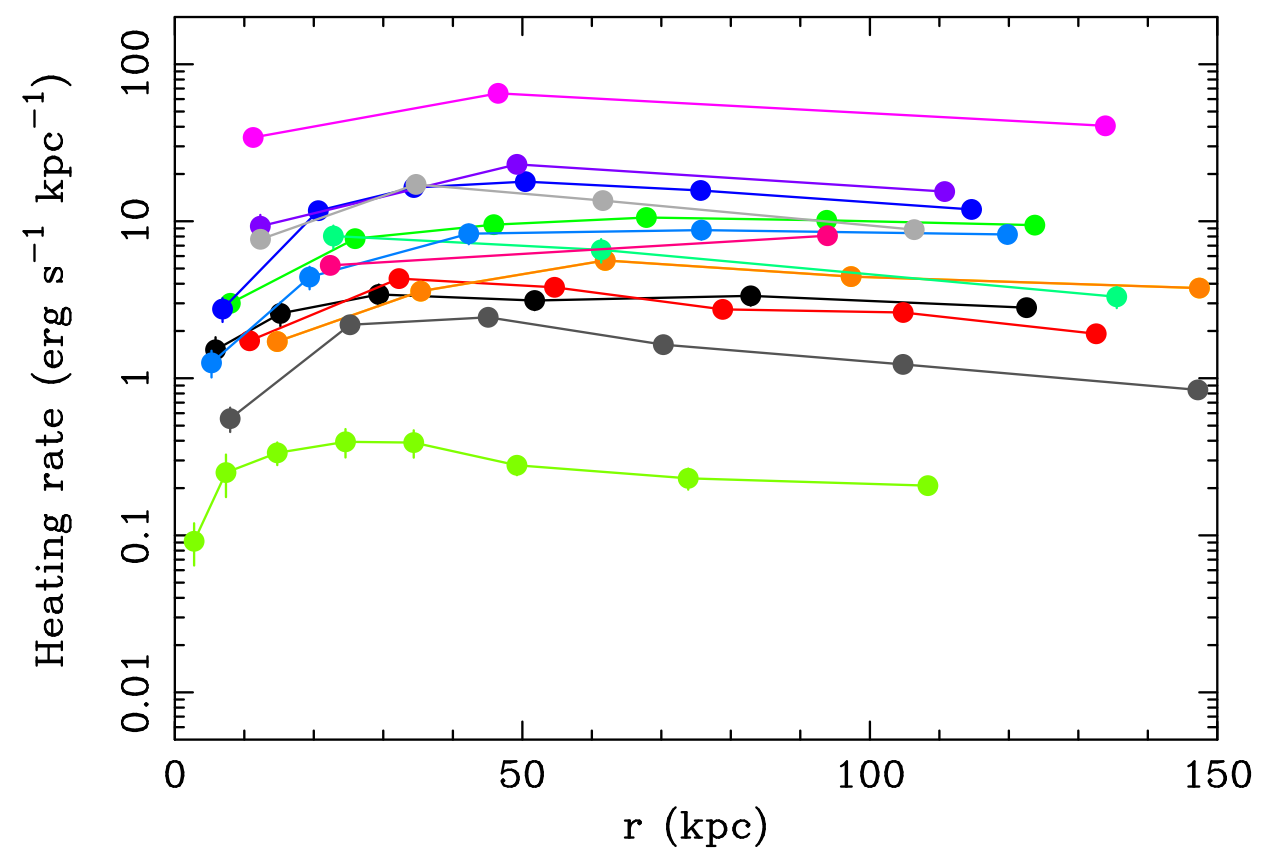

Fig. 19. The instantaneous heating rate required as a function of radius for a sample of massive cooling-flow clusters. These results indicate that the heating has to be spatially distributed over a substantial physical volume. Figure adapted from Voigt \& Fabian (2004).

the outer bubbles in the Perseus cluster supports this hypothesis Fabian et al. 2002).

The bubbles discussed so far have radii from about 1 to $15 \mathrm{kpc}$. Recently, giant bubbles of radius $100 \mathrm{kpc}$ have been found $100-200 \mathrm{kpc}$ from the centers of the Hydra-A nucleus (Nulsen et al. 2004) and in MS0735.6+7421 (McNamara et al. 2005), and some bubbles with little energy (e.g. Blanton et al. (2004)). The minimum energy of a bubble can be estimated from its volume $V$ and surrounding pressure $P$ as $E_{\text {bubble }}=P V$. If the interior pressure is entirely relativistic in gas with a ratio of specific heats of $4 / 3$ then $E_{\text {bubble }}=4 P V$. The energies are significant for typical bubbles (Birzan et al. 2004) and exceed $10^{61}$ erg for the giant bubbles.

\subsubsection{The simple theory of bubbles}

Bubbles from radio sources were first predicted by Gull \& Northover (Gull \& Northover 1973) and studied analytically before the Chandra/XMM-Newton era by Churazov et al. (2000, 2001) (see also Soker et al. 2002) and simulated by Heinz et al. (1998). They are inflated by jets from the nucleus and typically form in pairs either side of the nucleus and seemingly attached to the nucleus. If a jet of power $L$ steadily inflates a bubble of radius $R$ then, after an initial supersonic phase and assuming radiative losses are negligible, the bubble expansion with time $t$ follows from the conservation of energy as $L t=P V \propto P R^{3}$. Thus $R \propto t^{1 / 3}$ 
and the expansion speed of the bubble $v_{\text {bubble }} \propto t^{-2 / 3}$. The bubble is of much lower density than its surroundings so is buoyant and rises at a velocity close to the local gravitational free-fall value $v_{\text {grav }}$. When $v_{\text {bubble }}$ drops below $v_{\text {grav }}$ the bubble detaches from the jet and rises. A new bubble then forms if the jet remains powered.

A simple estimate of the power injected into the bubbles can be obtained by dividing $E_{\text {bubble }}$ by an estimate of the age of the bubble, which can be obtained from the above scaling (say, $R / v_{\text {grav }}$ ) (Birzan et al. 2004; Dunn \& Fabian 2004; Dunn et al. 2005). These are typically several orders of magnitude higher than the inferred radio luminosity of the jets and nucleus showing that the radio luminosity is a poor guide to the power of a jet. Such radio jets and lobes can have a radiative efficiency (ratio of observed synchrotron luminosity to mechanical power) as low as $10^{-3}$ or even $10^{-4}$. This, together with time variability of the jets, indicates that the very poor correlation between the radio power of the central source in a cluster and the heating power (Voigt \& Fabian 2004; Kaastra et al. 2004) does not necessarily imply that the mean jet power does not correlate well.

The power of the jets is mostly sufficient to offset radiative cooling in the cluster core provided it is steady. Birzan et al. (2004) did however note that it is insufficent in some objects. This could indicate that radio source heating is not a general process or that it can vary by factors of a few on the bubbling timescale, which ranges between $\sim 5 \mathrm{Myr}$ and $\sim 50 \mathrm{Myr}$. This timescale is still shorter than the inner radiative cooling timescale which is typically 100-300 Myr.

A further problem they raise is that bubbles were only found in 10 per cent of the clusters they examined, so they either do not occur in many clusters or if they do then they do not occur often. This result could however be a selection effect from the examination of a sample of clusters mixed in type (i.e. whether the cooling flow problem applies to that cluster or not) and signal-to-noise. A simple inspection by ACF of Chandra images of the 30 per cent of the 55 brightest clusters in the Sky listed by Edge et al. (1992) which have central $t_{\text {cool }}<5$ Gyr shows that all but one have clear bubbles, so we suspect that bubbles are indeed common enough to be a viable ingredient in the solution to the cooling flow problem. A more detailed analysis of the issue by Dunn et al. (2005) shows that at least 75 per cent of the cluster cores needing heating have bubbles. This means that the duty cycle of bubbles is such that they are (detectably) present for 75 per cent of the time.

Finally, before examining numerical simulations and then the heating mechanism in detail, we note that the total energy required to stave of cooling for several Gyr in a luminous cluster implies a large central black hole mass (Fabian et al. 2002; assuming a mass-to-energy conversion effiency of 10 per 
cent). Fuiita \& Reiprich (2004) compared black hole masses predicted by the velocity dispersion relation (Tremaine 2002) and found that they often fall short of what is required. However for the very few systems where the mass has been measured (e.g. M87) there is no problem. Perhaps the conclusion here would be that massive black holes in cluster cores lie above the $M_{\mathrm{BH}}-\sigma$ relation for lower mass galaxies.

\subsubsection{Numerical simulations of radio bubbles}

Many groups have now carried out numerical hydrodynamical simulations of the behaviour of bubbles in the ICM (Brüggen \& Kaiser 2001; Quilis et al. 2001; Brüggen \& Kaiser 2002; Brüggen 2003; Basson \& Alexander 2003; Omma \& Binnev 2004; Omma et al. 2004; Ruszkowski \& Begelman 2002; Ruszkowski et al. 2004a,b; Robinson et al. 2004; Vecchia et al. 2004; Revnolds 2005).

Most have been 2D or 3D and use the FLASH or ZEUS codes (see Gardini \& Ricker 2004) for a brief discussion and comparison of many of the simulations). Several assume that the surrounding gas is isothermal. Many produce bubbles which are very unstable (to Rayleigh-Taylor and Kelvin Helmholtz instabilities depending on the motion of the bubble at the start of a run) and collapse once they have risen more than their own height, which is most unlike the observed bubbles. Indeed most simulated bubbles do not look like the observed ones.

Several of the early simulations relied on the dragging out of cooler gas from the smallest cluster radii by the bubbles as the explanation of why so little cool gas is seen in real clusters. This explanation however only works over the lifetime of the cluster (say 5 Gyr) if the cooler gas is dragged out beyond the cooling radius (i.e well beyond $100 \mathrm{kpc}$ ), otherwise it either falls back in or mixes in and reduces the cooling time of gas just beyond the center. A catastrophic cooling flow is only being postponed for a while. Although this explanation can work temporarily it cannot provide a comprehensive solution.

When heating is estimated in simulations it is often unclear what it is due to. Some work is done by the buoyant bubbles rising and the sinking of cooler gas. This is the heating mechanism of the 'effervescent heating' approach of Begelman (2001) (see also Ruszkowski et al. 2004a). $P d V$ work is done as the bubbles are made in the first place. That energy presumably propagates as a sound wave and need not be dissipated locally unless shocks are involved or the ICM is viscous.

Heating by a mixture of weak shocks (at small radii) and viscous damping of sound waves (at larger radii) has been proposed by Fabian et al. (2003b) on the basis of the ripples seen in deep Chandra images of the Perseus cluster (see also Forman et al 2004 for a discussion of ripples in the Virgo cluster). 
Viscosity has been included in simulations by Ruszkowski et al. (2004a, b) and by Reynolds et al. (Reynolds 2005) and appears to dissipate 20-30 per cent of the $P d V$ energy from the bubbles. Transport of the energy by sound waves and dissipating it over a lengthscale of 50-100 kpc provides a fairly gentle and distributed source of heat. The bubbles in a viscous medium also better resemble the observed ones (Revnolds 2005).

Fuiita \& Suzuki (2005) and Mathews et al. (2005) argue that strong sound waves will shock and heat the innermost region and not deposit energy further out where it is needed. However a very deep image of the Perseus cluster (Fabian et al. 2005b) shows that the shock (Fig. 15) is isothermal. This may indicate that thermal conduction operates at least within the inner parts of the cool region to share the energy released by the bubbling process.

\subsection{Is the ICM turbulent?}

Several workers (Cho et al. 2003; Inogamov \& Sunvaev 2003; Chandran 2004; Fuijta \& Reiprich 2004) suggest that the ICM is turbulent. Such motions can transport heat and the dissipation of the turbulence is itself a source of heat. Schuecker et al. (2004) have measured brightness and temperature fluctuations in the Coma cluster (which is not a cooling flow cluster). Vogt \& Ensslin (2004) have determined a limited turbulent-like spectrum in the Faraday Rotation Measures of the Hydra cluster core. Ensslin (2005) have extended their work to predict velocities and length-scales for the turbulence in a number of cool core clusters. They argue that a small-scale turbulent dynamo is maintaining magnetic fields and that turbulent dissipation can balance radiative cooling.

Contrary indications have been identified (Fabian et al 2003b) in the highly extended, and often linear, optical filaments in the Perseus (Conselice et al. 2000) and other clusters (e.g A1795, Cowie et al. 1983; Centaurus, Crawford et al. 2005). Such filaments have coherent velocity fields and small velocity widths ( $\mathrm{Hu}$ et al. 1985; Crawford et al. 2005; Hatch et al 2005b) so must be relatively old (perhaps $100 \mathrm{Myr}$ ) and in thermal pressure equilibrium with the surrounding ICM. The optical surface brightness of such filaments translates to a thickness of the emission regions of less than a pc. If the ICM is turbulent and pushing on a filament, it will respond after interacting with its own column density which means a kpc or so of ICM, given the thousandfold higher density in the filament. This is only a few arcsec in nearby clusters so the apparent linear nature of the filaments would soon be destroyed by a fully turbulent ICM. They may however reflect streamlines and could have been drawn out by the motion of bubbles. Note the similarity of the horseshoeshaped filament in the Perseus cluster to that of streamlines behind rising 
water bubbles; see Fig. 184 in van Dvke 1982 and discussion by Fabian et al. $(2003 \mathrm{~b})$

The inner ICM in clusters with significant linear filaments may therefore have subsonic random flows, particularly radial behind bubbles. Whether this is turbulence depends on how turbulence is defined. It does not resemble fully-

developed hydrodynamical turbulence (see Fig. 186 in van Dvke 1982) but is not dissimilar from flows in some magnetohydrodynamical simulations (e.g. Schekochihin et al. 2004).

What is unclear is the driving mechanism for turbulence in a cluster core. A subcluster merger may well have made the Coma cluster ICM turbulent, but such mergers will not have strongly affected the dense core cluster under discussion here (but see Fuiita \& Reiprich 2004). Driving turbulence into the dense core will require considerable energy. Rising radio bubbles are a possible driver but whether their motion leads to a turbulent cascade of energy to much smaller scales must be determined. The viscosity of the ICM may play a key role in this issue.

The abundance gradients seen at the centers of many cool core clusters provides a further constraint on turbulence and diffusion. Rebusco et al. (2005) have modelled the production and maintenance of the gradient in the Perseus cluster and find a diffusion coefficient $D \sim v \ell \approx 2 \times 10^{29} \mathrm{~cm}^{2} \mathrm{~s}^{-1}$. Graham et al. (2005) have carried out a similar analysis on the more abundance-peaked Centaurus cluster to obtain a value of $D \sim 5$ times smaller.

\subsection{Multiphase flows}

Large scale global flows have been considered that transfer significant thermal energy from one region of the cluster to another. Mathews et al. (2003, 2004) have discussed flows which move in both radial directions. To be long lived, both mass and energy must be supplied to the inflowing gas over a large volume. The energy is assumed to be derived from bubbles.

Fabian et al. (2003a) considered a multiphase flow in which outer denser blobs fall inward and mix with inner, cooler blobs, thereby tapping the extended gravitational potential. Unless there is some outflow or cooling, however, mass builds up near the cluster center. 


\subsection{Role of Magnetic Fields and Cosmic Rays}

A cooling flow will amplify tangled magnetic fields in the intracluster gas (Soker \& Sarazin 1990). Magnetic fields in the general ICM have been found through radio observations of Faraday Rotation (Kim et al. 1991; Clarke et al. 2004). Such observations also show it rising in cool core clusters where the highest values have been found (Carilli \& Tavlor 2002). The results indicate a roughly cellular structure for the magnetic field of a few kpc (e.g. the Centaurus cluster, Tavlor et al. 2002). Such a structure may occur from a spectrum of magnetic fields perhaps due to turbulence (Ensslin 2005). Near the center the magnetic pressure can be 10 per cent of the thermal pressure, perhaps more. An important effect of the magnetic field is that it can dramatically reduce the microphysical transport processes in the ICM.

Cosmic rays are a likely additional component in cluster cores. Relativistic electrons produce the minihalo in the X-ray peaked region of the Perseus cluster (see Gitti et al. 2004 for a theoretical discussion) and may produce a hard X-ray component by inverse Compton scattering (Sanders et al. 2005). The effects of cosmic rays could be important in mediating the flow of mechanical energy in the core.

Cen (2005) notes that a cosmic ray phase can make heated gas thermally stable. A floor temperature of 0.3 times the local ambient temperature is derived if the cosmic ray pressure is $1 / 3$ of the ambient value. This situation is different from the similar factor seen in observations, which applies across the whole cool region, not just locally. Note that gas is not observed to "pile up" at any particular temperature.

If cosmic rays are spread through the core in many small bubbles, then they would be very effective in dissipating the energy in sound waves (Heinz \& Churazov 2005). Such bubbles must be small not to be detectable in deep images (eg. Fabian et al 2005b).

\subsection{Feedback}

If AGN heating balances cooling on timescales of $\sim 10^{8}$ to a few $10^{9} \mathrm{yr}$ then some feedback is needed to prevent either a cooling catastrophe (none has yet been found) or an event heating all the central gas and blowing it out. Bondi accretion onto a central black hole provides a link between the two regions but the radius range from the Bondi radius of say $50 \mathrm{pc}$ to the ICM at $50 \mathrm{kpc}$ is a factor of one thousand. The volume occupied by the black hole and its accretion radius which is required to provide the heating is one part in a billion! 
Such numbers emphasize the problem faced by the necessary feedback. Some attempts to model feedback are discussed by Ciotti \& Ostriker (2001); Kaiser \& Binney (2003): Nulsen et al. (2004), Soker \& Pizzolato (2005), Omma \& Binnev (2004); Binnev (2004); Hoeft \& Brüggen (2004); (Churazov et al. 2005);(Pizzolato \& Soker 2005). The common occurrence of star formation in the central galaxy and also of giant bubbles shows that any feedback need not be perfect on timescales less than $10^{8}$ yr. Donahue et al. (2005) study two clusters which show little central temperature drop or central radio source yet have central cooling times of $\sim 1$ Gyr. Such clusters, which are in the minority, may be recovering from giant outbursts.

\subsection{Other heat sources and mechanisms}

Cosmic ray heating has been invoked in several models (Colafancesco et al. 2004; Totani 2004). Generally to be effective, the pressure in cosmic rays would have to exceed that of the thermal gas (Loewenstein 1991). Excess ionization by suprathermal electrons could change the spectral appearance of gas in a cooling flow. This has been studied by $\mathrm{Oh}$ (2004), who finds that such electrons provide much more heating than ionization, so reverting to the problems raised above by Loewenstein (1991).

Heating by galaxy motions have been revived by El-Zant et al. (2004) (see also Faltenbacher et al. 2005) following early work by Miller (1986). It may be a viable distributed source of heat if the mass-to-light ration of the galaxies passing through the inner regions exceeds 10 (but see Kim 2005).

Dark matter interactions have been discussed by several authors Qin \& Wu 2001; Totani 2004; Chuzhov \& Nusser 2004). Similarities with the solar corona have been discussed by Makishima (2001) and Kaastra et al. (2004). Brüggen \& Ruszkowski (2005) discuss that viscous heating, if it occurs in real MHD plasmas, could provide significant heating during structure formation.

One last possibility is that sedimentation acts (Fabian \& Pringle 1977). Helium then accumulates at the cluster center (Gilfanov \& Sunvaev 1984) possibly explaining the puzzling low metallicity found at the center of some nearby clusters observed with Chandra. The main low temperature cooling gas is then helium rich which can produce much weaker emission lines. Stars formed from helium rich gas will have short lifetimes so avoiding some constraints on the cold mass sink (Fabian et al. 2003c; Ettori \& Fabian 2005). Tangled magnetic fields will of course significantly reduce the prospect of any sedimentation. 


\section{Discussions}

The simplest explanation for the common appearance of cold core, X-ray peaked clusters is that, when averaged over tens of Myr, the radiative cooling is balanced in part by distributed heating. Thermal conduction as a means of distributing heat from outer gas is ruled out for low and intermediate temperature clusters. It may however have a role in spreading the energy in the central parts. A plausible mechanism is the dissipation of energy propagating through the ICM from a central radio source. Such a process stems massive cooling onto the BCG which would otherwise gain a total stellar mass $\gg 10^{12} \mathrm{M}_{\odot}$. The process is therefore a vital ingredient in stopping the growth

of the most massive galaxies (Fabian et al. 2002b; Benson et al. 2003; Binney 2004). NOte that most semi-analytic models for galaxy formation (e.g. Kauffmann et al 1999) already needed to suppress cooling in massive haloes in order to match observation.

Difficulties and doubts remain with regard to the issues of the energy dissipation and distribution processes which are tied in with the transport processes in the gas. Similarly, it is not clear how the feedback manages to produce such similar cooling time profiles in systems where temperatures and thus masses differ by over an order of magnitude? There still remains the possibility that some process not yet foreseen, or at least not well studied will eventually prove more important than the effect of the central radio source, or will at least be important in mediating its effect. The central radio source is so common and so energetic however that it must at least be part of the solution. Similarly, the motions of galaxies or interacting dark matter, if it exists, could be important in heating cluster cores along with the AGN. Given the wide range of objects in which a balance is required, we suspect that a single mechanism is dominant, rather than several.

The need for a heating-cooling balance is in a time-averaged sense, over intervals of about $10^{8} \mathrm{yr}$. In most cases the heating has not been so energetic as to drive gas out of the inner regions nor so weak as to allow much cooling at very high rates. Examples of objects at the extremes are Hydra A and Cygnus A where heating is high (but is dumped mostly at large radii outside the cool region) and A2597 and RXC1504.1-0248 where cooling appears to be high (in the latter object over 70 per cent of the total X-ray luminosity emerges from the cool core; Böhringer et al. 2005).

In most objects residual cooling at a rate of about 10 per cent of the simple unheated cooling rates appears to occur. It could be larger if non-radiative cooling, due to mixing say, is occurring. Stars form from the cooled gas giving the excess blue light seen in the BCGs. Mass loss from such stars can make the cooled gas dusty and radio bubbles drag some of it back out to large radii. 
The time evolution of the heating / cooling balance is little understood. We suspect that the common temperature drop associated with the short central cooling times is due to radiative cooling and that heating only came into balance when the overall temperature structure was in place. Perhaps the central galaxy and its central $\mathrm{BH}$ grew until balance was achieved, and growth required cooling. Comparison of samples of clusters at $\bar{z}=0.22$ with $\bar{z}=$ 0.056 (Bauer et al. 2005) shows little surprisingly change in the distribution of cooling times at $50 \mathrm{kpc}$. The results imply that any balance was established well beyond $z \sim 0.3$.

Few massive cool core clusters are known at much higher redshifts than that of RXJ1347 at $z=0.44$. This may however be a selection effect. They will be absent from X-ray cluster samples if the central $\mathrm{BH}$ is a bright X-ray source such as a quasar. If a central quasar outshines the host cluster in Xrays then the object will generally be classified as a quasar. $\mathrm{H} 1821+643$ is a good example of a bright quasar in an X-ray peaked cluster at intermediate redshift $(z=0.297$, Fang et al. 2002). Searches for clusters around powerful radio-loud quasars and galaxies have found some examples at $z=0.5-1.1$ (Worrall et al. 2001; Crawford \& Fabian 2003; Siemiginowska et al. 2005) but no complete searches have been done. 


\section{Future work}

Further detailed deep studies with Chandra and XMM-Newton as well as future studies with Constellation-X and Xeus are needed in order to better understand the heating/cooling balance. There is considerable potential for studies of the cool and warm gas and dust around the BCG using HST, Spitzer and ground-based telescopes. Larger cluster samples are needed, particularly at medium to high redshifts. Determining the extent of a heating-cooling balance (or not) in groups, elliptical galaxies, and galaxy bulges is also important as is the evolution of any balance in all massive objects. 


\section{Acknowledgments}

We thank Mateusz Ruszkowski, Mitch Begelman, Roger Blandford, and an anonymous referee for a careful reading of this manuscript. ACF thanks the Royal Society for support. JRP was supported by a grant from NASA for scientific and calibration support of the RGS at Stanford. This was also supported by the U.S. Department of Energy under contract number DE-AC0276SF00515. 


\section{References}

Allen, S. W. et al. 1993, MNRAS 262, 901.

Allen, S. W. \& Fabian, A. C. 1997, MNRAS 286, 583.

Allen, S. W., DiMatteo, T., \& Fabian, A. C. 2000, MNRAS 311, 493.

Allen, S. W. et al. 2001, MNRAS 322, 589.

Allen, S. W. et al. 2001, MNRAS 324, 842.

Allen, S. W. et al. 2001, MNRAS 328, 37.

Arnaud, K. A. Astronomical Data Analysis Software and Systems V ASP Conference Series, Vol. 101, 1996, George H. Jacoby and Jeannette Barnes, eds.

Arnaud, K. A. 1988, "Cooling Flows in Clusters and Galaxies", Fabian, A. C. ed., Dordrecht: Kluwer.

Arnaud, M. \& Raymond, J. 1992, ApJ 398, 394.

Arnaud, M. \& Rothenflug, R. 1985, A \& AS 60, 425.

Bailey, M. E. 1982, MNRAS 201, 271.

Balbus, S. A. 1988, ApJ 328, 395.

Balbus, S. A., \& Soker, N. 1989, ApJ 341, 611.

Balbus, S. A. 1991, ApJ 372, 25.

Bar-Shalom, A., Klapisch, M., \& Oreg, J 2001, JQSRT 71, 169.

Basson J.F. \& Alexander P. 2003, MNRAS 339, 353.

Bayer-Kim, C. M. et al. 2002, MNRAS 337, 938.

Bauer, F. E. et al. 2002, AJ 123, 1163.

Bauer, F. E. et al. 2005, MNRAS 359, 1481.

Begelman, M. 2001, ASP conf. ser. 240, Gas \& Galaxy Evolution, ed. Hibbard, Rupen, \& van Gorkom, (San Francisco: ASP), 363.

Benson, A. J. et al. 2003, ApJ 599, 1.

Berrington, K. A., Eissner, W. B., \& Norrington, P. H. 1994, CoPhC 92, 290.

Bertschinger E., Meiksin A., 1986, ApJ, 306, L1

Binney, J. \& Cowie, L. L. 1981, ApJ 247, 464.

Binney, J. \& Tabor, G. 1995, MNRAS 276, 663.

Binney J. 2004, MNRAS 347, 1093.

Birzan, L., Rafferty, D. A., McNamara, B. R., Wise, M. W., \& Nulsen P. E. J. 2004, ApJ 607, 800.

Blanton E.L., Sarazin C.L., McNamara B.R., Wise M.W. 2001, ApJ 558, L15.

Blanton, E. L., Sarazin, C.L., McNamara, B.R., Clarke, T.E., 2004, ApJ, 612, 817

Böhringer, H. \& Hensler, G. 1989, A \& A 215, 147.

Böhringer, H. et al. 1993, MNRAS 264, 25.

Böhringer, H. et al. 2001, A \& A 365, 181.

Böhringer, H., Matsushita, K., Churazov, E., Ikebe, Y., \& Chen, Y. 2002, A\&A 382, 804.

Böhringer, H. et al. 2005, astro-ph/0507431.

Branduardi-Raymont, G. et al. 1981, ApJ 248, 55. 
Brighenti F., Mathews W.G., 2003, ApJ, 587, 580 .

Bregman, J. N. \& David, L. P. 1988, ApJ 326, 639.

Bregman, J., Fabian A.C., Miller E.D., Irwin J.A., 2005, ApJ submitted.

Brown, G. V. et al. 1998, ApJ 502, 1015.

Brown, G. V. et al. 2002, ApJS 140, 589.

Brüggen, M. \& Kaiser, C.R. 2002, MNRAS 325, 676.

Brüggen, M. \& Kaiser, C.R. 2002, Nature 418, 301.

Brüggen, M. 2003, ApJ 592, 839.

Brüggen, M. \& Ruszkowski 2005, astro-ph/0512148

Buote, D. A., \& Fabian, A. C. 1998, MNRAS 296, 977.

Buote, D. A. et al. 1998, MNRAS 309, 685.

Buote, D. A. et al. 2000, MNRAS 311, 176.

Buote, D. A. et al. 2000, ApJ 544, 242.

Buote, D. A., Lewis, A. D., Brighenti, F., Mathews, W. G. 2003, ApJ 594, 741.

Burns, J. O. 1990, AJ 99, 14.

Canizares, C. R., Clark, G. W., Markert, T. H., Berg, C., Smedira, M., Bardas, D., Schnopper, H. \& Kalata, K. 1979, ApJ 234, L33.

Canizares, C. R., Clark, G. W., Jernigan, J. G., \& Markert, T. H. 1982, ApJ 262, L32.

Carilli, C. L. \& Taylor, G. B. 2002, ARA\&A 40, 319.

Carter, D., Johnstone, R. M., \& Fabian, A. C. 1997, MNRAS 285, L20.

Cen, R. 2005, ApJ 620, 191.

Chandran, B. \& Cowley, S. C. 1998, PhRvL 80, 3077.

Chandran, B. D. G. 2004, ApJ 616, 169.

Chen, H. et al. 2005, ApJ 618, 1086.

Cho J., Lazarian A., Honein A., Knaepen B., Kassinos S. \& Moin P. 2003, ApJ 589, L77.

Cho, J. \& Lazarian, A. 2004, JKAS 37, 557.

Churazov, E., Forman, W., Jones, C., Böhringer, H. 2000, A \& A 356, 788.

Churazov E., Brüggen M., Kaiser C.R., Böhringer H., Forman W., 2001, ApJ, 554,261

Churazov, E., Forman, W., Jones, C., Böhringer, H. 2003, ApJ 590, 225.

Churazov, E., Sazonov S., Sunyaev R., Forman W., Jones C., Böhringer H., 2005, MNRAS, 363, L81

Chuzhoy, L. \& Nusser, A. 2004, astro-ph/0408184.

Ciotti, L. \& Ostriker, J. P. 2001, ApJ 551, 131.

Clarke, T. E., Blanton E. L., \& Sarazin, C. L. 2004, ApJ, 616, 179

Clarke, T. E., Sarazin, C.L., Blanton E.L., Neumann D.M., Kassim N.E., 2005, ApJ, 625, 948

Colafrancesco, S., Dar, A., De Rujula, A. et al. 2004, A \& A 413, 441.

Conselice, C.J., Gallagher J.S., Wyse R.F.G. 2000, ApJ 559, 79.

Cowie, L. L., Fabian, A. C., \& Nulsen, P. E. J. 1980, MNRAS 191, 399.

Cowie, L. L., Hu, E. M., Jenkins, E. B., \& York, D. G. 1983, ApJ 272, 29.

Crawford C.S., Allen S.W., Ebeling H., Edge A.C., Fabian A.C., 1999, MN- 
RAS, 306, 875.

Crawford C. S. \& Fabian, A. C. 2003, MNRAS 339, 1163.

Crawford, C. S., Hatch, N. A., Fabian, A. C., \& Sanders, J. S. 2005, astroph/050726.

David, L. P. et al. 1993, ApJ 412, 479.

David, L. P. et al. 2001, ApJ 557, 215.

Domainko, W. et al. 2004, A\&A 427, 9.

Donahue, M. et al. 2000, ApJ 569, 689.

Donahue, M., Voit G.M., O’Dea C.P., Baum S.A., Sparks W.B., 2005, ApJ, 630, L13

Donahue, M. et al. 2005, ApJ 630, 13.

Dunn, R. J. H. \& Fabian, A. C. 2004, MNRAS 355, 862.

Dunn, R. J. H., Fabian A.C., Taylor G.B., 2005, MNRAS, in press (astro-ph/0510191)

Edge, A. C. \& Stewart, G. C. 1991, MNRAS 252, 414.

Edge, A. C., Stewart, G. C., Fabian, A. C. 1992, MNRAS 258, 559.

Edge, A. C. 2001, MNRAS 328, 762.

Elwert, G. 1952, Z. Naturf. 7a, 432, 703.

El-Zant, A. A. 2004, MNRAS 354, 169.

Ensslin, T. A. \& Vogt, C. 2005, astro-ph/0505517

Ettori, S., Fabian, A. C., Allen, S. W., \& Johnstone, R. M. 2002, MNRAS 331, 635.

Ettori, S., Fabian A.C., 2005, MNRAS submitted

Fabian, A. C. et al. 1974, ApJL 189, L59.

Fabian, A. C., Pringle, J. E., \& Rees, M. J. 1976, MNRAS 175, 43.

Fabian, A. C. \& Pringle J. E. 1977, MNRAS 181, 5.

Fabian, A. C., Hu, E. M., Cowie, L. L., \& Grindlay, J. 1981, ApJ 248, 47.

Fabian, A. C., Nulsen, P. E. J., Canizares, C. R. 1982, MNRAS 201, 933.

Fabian, A. C., Nulsen, P. E. J., \& Canizares, C. R. 1984, Nature 310, 733.

Fabian, A. C. et al. 1985, MNRAS 216, 923.

Fabian, A. C. et al. 1994, ApJ 436, L63.

Fabian A. C. et al., 2000, MNRAS, 318, L65

Fabian A.C., Mushotzky R.F., Nulsen P.E.J., Peterson J. R., 2001, MNRAS, 321, L20

Fabian, A. C., Celotti, A., Blundell, K. M., Kassim, N. E., Perley, R. A. 2002, MNRAS, 331.

Fabian A.C., Allen, S.W., Crawford, C.S., Johnstone R.M., Morris, R.G., Sanders J.S., Schmidt R.W., 2002a, MNRAS, 332, L50

Fabian, A. C., Voigt, L. M., \& Morris, R. G. 2002b, MNRAS 335, 71.

Fabian, A. C. et al. 2003, MNRAS 344, 43.

Fabian A. C. et al. 2003, MNRAS 344, 48.

Fabian, A. C., Sanders, J.S., Crawford C.S., Ettori, S., 2003c, MNRAS, 341, 729.

Fabian, A. C., Celotti, A., Johnstone, R.M., 2003, MNRAS, 338, L7.

Fabian, A. C. et al. 2005a, astro-ph/051222. 
Fabian, A C. et al. 2005b, MNRAS 360, L20.

Fang, T., Bryan, G., \& Canizares, C. 2002, ApJ 564, 604.

Faltenbacher, A. et al. 2005, MNRAS 358, 139.

Forman, W. et al. 2003, astro-ph/0312576.

Friaca, A. C. S. 1986, A \& A 164, 6.f

Field, G. B. 1965, ApJ 142, 531.

Fujita, Y. \& Reiprich, T. H. 2004, ApJ 612, 9.

Fujita, Y., Susuki T.K., 2005, ApJ, 630, L1

Fukazawa, Y. et al. 1998, PASJ 50, 187.

Fukazawa, Y. et al. 2000, MNRAS 313, 21.

Gardini \& Ricker 2004, Mod Phys. Lett. A19.

Gilfanov, M. R. \& Sunyaev, R. A. 1984, PAZh 10, 329.

Gilfanov, M. R., Sunyaev, R. A., Churazov, E. M., 1987, SvAL 13, 3.

Gitti, M. et al. 2004, A\&A, 417, 1.

Goldreich, P. \& Sridhar, S. 1995, ApJ 438, 763.

Gorenstein, P. et al. 1977, ApJL 216, L95.

Graham et al. 2005, in preparation.

Gu, M. F. et al. 1999, ApJ 518, 1002.

Gu, M. F. et al. 2003, ApJ 582, 1241.

Gull, S. F., \& Northover, K. J. E. 1973, Nature 244, 80.

Hatch, N. A. et al. 2005a, MNRAS 358, 765.

Hatch, N. A. et al. 2005b, MNRAS, submitted

Heinz, S., Reynolds, C. S., \& Begelman, M. C. 1998, ApJ 501, 126.

Heinz, S., Churazov E., 2005, ApJ, 634, L141

Helmken, J. et al. 1978, ApJL 221, L43.

Hicks, A. \& Mushotzky 2005, astro-ph/0508143.

Hoeft, M. \& Brüggen, M. 2004, ApJ 617, 896.

Horner, D. J., Mushotzky, R. F., \& Sharf, C. A. 1999, ApJ 520, 78.

Hu, E. M., Cowie, L. L., \& Wang, Z. 1985, ApJS 59, 447.

Inogamov, N. A. \& Sunyaev, R. A. 2003, Ast. Lett. 29, 791.

Ikebe, Y. et al. 1997, ApJ 481, 660.

Jaffe, W., Bremer, M. N., \& van der Werf, P. P. 2001, MNRAS 324, 443.

Jaffe,, W., Bremer, M. N., \& Baker, K. 2005, MNRAS 360, 748.

Johnstone, R. M. et al. 1992, MNRAS 255, 431.

Johnstone, R. M., Allen, S. W., Fabian, A. C., Sanders, J. S., 2001, MNRAS $336,299$.

Jones, C. \& Forman, W. 1984, ApJ 276, 38.

Jordan, C. 1969, MNRAS 142, 501.

Kaastra, J. S. 1992, Internal SRON-Leiden Report.

Kaastra, J. S., Mewe, R., \& Nieuwenhuijzen, H. 1996, in UV and X-ray Spectroscopy of Astrophysical and Laboratory Plasmas, pg 411., K. Yamashita and T. Watanabe. Tokyo: Universal Academy Press.

Kaastra, J. S., Ferrigno, C., Tamura, T., Paerels, F. B. S., Peterson, J. R., \& Mittaz, J. P. D. 2001, A \& A 365, 99.

Kaastra, J. S. et al. 2003, A \& A 413, 415. 
Kaiser C.R. \& Binney, J. 2003, MNRAS 338, 837.

Kim, K.-T., Kronberg, P. P., \& Tribble, P. C. 1991, 379, 80.

Kim W.-T. \& Narayan, R. 2003a, MNRAS 596, L139.

Kim, W.-T. \& Narayan, R. 2003b, MNRAS 596, 889.

Kim W.-T., Amr A. E., \& Kaminokowski, M. 2005, ApJ accepted.

Lahav, O., Edge, A. C., Fabian, A. C., \& Putney, A. 1989, MNRAS 238, 881.

Lea, S. M., Silk, J., Kellogg, E., Murray, S. 1973, ApJL 336, L13.

Liedahl, D. A., Osterheld, A. L., \& Goldstein, W. H. 1995, ApJL, 438, 115.

Loewenstein, M. 1989, MNRAS 238, 15.

Loewenstein, M. 1990, ApJ 349, 471.

Loewenstein, M., Zweibel, E. G., \& Begelman, M. C. 1991, ApJ 377, 392.

Makishima, K. 2001, PASJ 53, 401.

Markevitch, M. 1998, ApJ 403, 77.

Markevitch, M. et al. 2000, ApJ 541, 542.

Mathews, W. G., Brighenti, F., Buote, D. A., Lewis, A. D. 2003, ApJ 596, 159.

Mathews, W. G., \& Brighenti, F., Buote D.A., 2004, ApJ, 615, 662

Mathews, W. G., Faltenbacher, A., \& Brighenti, F. 2005, ApJ accepted, astro-ph/0511151.

Matsumoto, H. 1996, PASJ 48, 201.

Mazzotta, P., Mazzitelli, G., Colafrancesco, S., \& Vittorio, N. 1998, A \& AS 133, 403.

McNamara, B. R. et al. 2000, ApJ 534, 135.

McNamara, B. R. et al. 2001, ApJ, 562, L149

McNamara, B. R. et al. 2004, ApJ 601, 173.

McNamara, B. R. et al. 2005, Nature 433, 45.

Miller, L. 1986, MNRAS 220, 713.

Mukai, K., Kinihabwala, A., Peterson, J. R., Kahn, S. M., \& Paerels, F. 2003, ApJ 586, 77.

Molendi, S. \& Pizzolato, F. 2001, ApJ 560, 194.

Malagoli, A., Rosner, R., \& Bodo, G. 1987, ApJ 319, 632.

Maron, J., Chandran, B. D., \& Blackman, E. 2004, PhRvL 92, 5001.

Mewe, R., Gronenschild, E. H. B. M., \& van den Oord, G. H. J. 1985, A \& AS 62, 197.

Mewe, R., Lemen,J. R., \& van den Oord, G. H. J. 1986, A \& AS 65, 511.

Mewe, R., Kaastra, J. S., Liedahl, D., 1995, Legacy vol. 6, 16.

Mewe, R. 1999, in X-ray Spectroscopy in Astrophysics, ed. J. van Paradijs, \& J. A. M. Bleeker (Berlin: Springer), 347.

Mitchell, R. J. et al. 1975, ApJL 200, L5.

Mitchell, R. J. et al. 1976, MNRAS 175, 29.

Morris R.G., Fabian A.C., 2003, MNRAS, 338, 824

Morris R.G., Fabian A.C., 2005, in preparation.

Navarro, J. F., Frenk, C. S., White, S. D. M. 1997, ApJ 490, 493.

Narayan, R., \& Medvedev, M. V. 2001, ApJ 562, 129.

Nevalainen, J., Markevitch, M., \& Forman, W. 2000, ApJ 532, 694. 
Nulsen, P. E. J. et al. 1982, MNRAS 199, 1089.

Nulsen, P. E. J. 1986, MNRAS 221, 377.

Nulsen, P. E. J., McNamara, B. R., Wise, M. W., \& David, L. P., astro-ph/0408315.

O’Dea C. P., Payne, H., \& Kocevski 1998, ApJ 116, 623.

Oegerle, W. R. et al. 2001, ApJ 560, 187.

Oh, S. P. 2004, MNRAS 353, 468.

Omma, H. \& Binney, J., MNRAS 350, 13.

Omma, H., Binney, J., Bryan, G., Slyz, A. 2004, MNRAS 348, 1105.

Pedlar, A. et al. 1990, MNRAS 246, 477.

Peres, C. B. et al. 1998, MNRAS 298, 416.

Peterson, J. R. et al. 2001, A \& A 365, 104.

Peterson, J. R. et al. 2003, ApJ 590, 207.

Peterson, J. R., Jernigan J. G., \& Kahn, S. M. 2004, ApJ 615, 545.

Pizzolato F., Soker N. 2005, ApJ, 632, 821

Ponman, T. J., Cannon, D. B., \& Navarro, J. F. 1999, Nature 397, 135.

Prestwich, A. H., Joy, M., Luginbuhl, C. B., Sulkanen, M., \& Newberry, M. 1997, ApJ 477, 144.

Qin, B. \& Wu, X. 2001, PRL 87, 1301.

Quilis V., Bower R.G., Balogh M.G., 2001, MNRAS, 328, 1091

Raymond, J. \& Smith, B. W. 1977, ApJS 35, 419.

Rebusco, P. et al. 2005, MNRAS 359, 1041.

Reynolds, C.S., McKernan, B., Fabian, A.C., Stone, J.M., Vernaleo, J.C., 2005, MNRAS, 357, 242

Robinson, K. et al., 2004, ApJ, 601, 621

Rosner, R. \& Tucker, W. H. 1989, ApJ 338, 761.

Ruszkowski M., Begelman M.C., 2002, ApJ 581, 223.

Ruszkowski, M., Brüggen, M., \& Begelman, M. C. 2004, ApJ 611, 158.

Ruszkowski, M., Brüggen, M., \& Begelman, M. C. 2004, ApJ 615, 681.

Sanders, J. S., Fabian, A. C., Allen S. W., \& Schmidt, R. W. 2004, MNRAS 349, 952.

Sanders, J. S., Fabian, A. C., \& Dunn, R. J. H. 2005, MNRAS 360, 133.

Sakelliou, I. et al. 2002, A\&A 391, 202.

Salome, P. \& Combes, F. 2004, A\&A 415, 1.

Schmidt, R. W., Allen, S. W., \& Fabian, A. C. 2001, MNRAS 327, 1057.

Shuecker, P., Finoguenov, A., Miniati, F., Böhringer, H., \& Briel, U. G. 2004, A \& A 426, 387.

Schekochihin, A. A. et al. 2004, ApJ 612, 276.

Siemiginowska, A. et al. 2005, ApJ, 632, 110

Silk, J. et al. 1986, ApJ 307, 1986.

Soker, N. \& Sarazin, C. L. 1990, ApJ 348, 73.

Soker, N., Blanton, E. L, \& Sarazin, C. L. 2002, ApJ 573, 533.

Soker, N., Blanton, E. L, \& Sarazin, C. L. 2002, A\&A 422, 445.

Soker, N. \& Pizzolato, F. 2005, ApJ 622, 847.

Sparks, W. B., Corollo, C. M., Macchetto, F. 1997, ApJ 486, 253. 
Sparks, W. B. et al. 2004, ApJ 607, 294.

Spitzer, L., Jr. 1962, Physics of Fully Ionized Gases (2nd ed.: New York: Interscience).

Stewart, G. C., Fabian, A. C., Jones, C., \& Forman, W. 1984, ApJ 285, 1.

Smith, R. K., Brickhouse, N. S., Liedahl, D. A., \& Raymond, J. C 2001, ApJ 556, 91.

Sutherland, R. S. \& Dopita, M. A. 1993, ApJS 88, 253.

Tabor, G. \& Binney, J. 1993, MNRAS 263, 323.

Takahara, M. \& Takahara, F. 1979, PThPh 62, 1253.

Tamura, T., Kaastra, J. S, Peterson, J. R. et al. 2001, A \& A 365, 87.

Tamura, T., Bleeker, J. A. M., Kaastra, J. S., Ferrigno, C. \& Molendi, S. 2001, A\&A 379, 101.

Tao, L. 1995, MNRAS 275, 965.

Taylor, G. B., Fabian, A. C., \& Allen, S. W. 2002, MNRAS 334, 769.

Thomas, P. A., Fabian, A. C., \& Nulsen P. E. J. 1987, MNRAS 228, 973.

Totani, T. 2004, PhRvL 92, 1301.

Tremaine, S. et al. 2002, ApJ 574, 740.

Tribble, P. C. 1989, MNRAS 238, 1247.

Tucker, W. H. \& Rosner, R. 1983, ApJ 267, 547.

Ulmer, M. P. \& Jernigan, J. G. 1978, ApJ 222, 85.

van Dyke, M. 1982, 'An Album of Fluid Motion', (Stanford: Parabolic Press). Vecchia, C. D. et al. 2004, MNRAS 355, 995.

Voigt L. M., Schmidt R. W., Fabian A. C., Allen S. W., Johnstone R. M., 2002, MNRAS, 335, L7

Voigt L. M. \& Fabian A. C. 2004, MNRAS, 347, 1130.

Voit, G. M. et al. 2003, ApJ 593, 272.

Voit, G. M., \& Bryan, G. 2001, Nature 414, 425.

Vogt, C. \& Ensslin, T. A. 2004, JKAS 37, 349.

White, D. A., Fabian, A. C., Johnstone, R. M., Mushotzky, R. F., Arnaud, K. A. 1991, MNRAS 252, 1991.

White, R. E. \& Sarazin, C. L. 1987, ApJ 318, 612.

White, R. E. \& Sarazin, C. L. 1987, ApJ 318, 621.

White, R. E. \& Sarazin, C. L. 1987, ApJ 318, 629.

White, R. E. \& Sarazin, C. L. 1988, ApJ 335, 688.

White, D. A., Jones, C., \& Forman, W. 1997, MNRAS 292, 419.

Wilman, R. J. et al. 2002, MNRAS 337, 63.

Wise, M. W. et al. 2004, ApJ 601, 184.

Worrall, D. M. et al. 2001, MNRAS 326, 1127.

Wu, K. K. S., Fabian, . C., \& Nulsen, P. E. J. 2000, MNRAS 318, 889.

Wu, X.-P., Xue, Y.-J., \& Fang, L.-Z. 1999, ApJ 524, 22.

$\mathrm{Xu}, \mathrm{H}$. et al. 1998, ApJ 500, 738.

Xu, H., Jin, G., \& Wu, X.-P. 2001, ApJ 553, 78.

$\mathrm{Xu}, \mathrm{H}$. et al. 2002, ApJ 576, 600.

Young, A. J., Wilson, A. S., \& Mundell, C. G. 2002, ApJ 579, 560.

Zakamska N.L., Narayan R., 2003, ApJ, 582, 162. 